\title{
Superconductivity in spin-3/2 systems: Symmetry classification, odd-frequency pairs, and Bogoliubov Fermi surfaces
}

\author{
Paramita Dutta $\odot$, Fariborz Parhizgar $\odot$, and Annica M. Black-Schaffer $\odot$ \\ Department of Physics and Astronomy, Uppsala University, Box 516, S-751 20 Uppsala, Sweden
}

(Received 28 June 2021; revised 26 August 2021; accepted 31 August 2021; published 17 September 2021)

\begin{abstract}
The possible symmetries of the superconducting pair amplitude is a consequence of the fermionic nature of the Cooper pairs. For spin-1/2 systems this leads to the $\mathcal{S P O T}=-1$ classification of superconductivity, where $\mathcal{S}, \mathcal{P}, \mathcal{O}$, and $\mathcal{T}$ refer to the exchange operators for spin, parity, orbital, and time between the paired electrons. However, this classification no longer holds for higher spin fermions, where each electron also possesses a finite orbital angular momentum strongly coupled with the spin degree of freedom, giving instead a conserved total angular moment. For such systems, we here instead introduce the $\mathcal{J P} \mathcal{T}=-1$ classification, where $\mathcal{J}$ is the exchange operator for the $z$ component of the total angular momentum quantum numbers. We then specifically focus on spin-3/2 fermion systems and several superconducting cubic half-Heusler compounds that have recently been proposed to be spin-3/2 superconductors. By using a generic Hamiltonian suitable for these compounds we calculate the superconducting pair amplitudes and find finite pair amplitudes for all possible symmetries obeying the $\mathcal{J P} \mathcal{T}=-1$ classification, including all possible odd-frequency (odd- $\omega$ ) combinations. Moreover, one of the very interesting properties of spin-3/2 superconductors is the possibility of them hosting a Bogoliubov Fermi surface (BFS), where the superconducting energy gap is closed across a finite area. We show that a spin$3 / 2$ superconductor with a pair potential satisfying an odd-gap time-reversal product and being noncommuting with the normal-state Hamiltonian hosts both a BFS and has finite odd- $\omega$ pair amplitudes. We then reduce the full spin-3/2 Hamiltonian to an effective two-band model and show that odd- $\omega$ pairing is inevitably present in superconductors with a BFS and vice versa.
\end{abstract}

DOI: 10.1103/PhysRevResearch.3.033255

\section{INTRODUCTION}

Properties of ordered matter are to a large extent set by the symmetry of the ordered state. For superconductivity, this means the symmetry of superconducting Cooper pairs. The symmetry of these Cooper pairs are classified following the antisymmetry of the Cooper pair wave function during exchange of all the quantum numbers between two constituent electrons, as a result of the fermionic nature of the electrons. This classification of superconducting pairs has become known as the $\mathcal{S P O T}=-1$ classification, with $\mathcal{S}, \mathcal{P}, \mathcal{O}$, and $\mathcal{T}$ referring to the exchange operators for spin, parity, orbital, and time, and is now well established in the literature [1,2].

Following this classification, Cooper pairs with spinsinglet and spin-triplet spin structures are further identified as odd-frequency (odd- $\omega$ ) or even-frequency (even- $\omega$ ) pairs, depending on their spatial parity and their symmetry under orbital index exchange between the electrons. Odd (even)- $\omega$ superconductivity refers to when the Cooper pair amplitude is odd (even) under the exchange of the time coordinates, or equivalently frequency, of the two constituent electrons,

Published by the American Physical Society under the terms of the Creative Commons Attribution 4.0 International license. Further distribution of this work must maintain attribution to the author(s) and the published article's title, journal citation, and DOI. as introduced by Balatsky and Abrahams [3] following the first prediction of odd-frequency order by Berezinskii in ${ }^{3} \mathrm{He}$ [4]. Finite odd- $\omega$ pair amplitudes have been predicted to exist mostly in superconducting hybrid structures [1,5-10], but also in a variety of other systems, such as multiband superconductors [11-13], topological materials [14-20], heavy-fermion compounds [21-23], and driven systems [24,25]. In several of these systems, experimental consequences of odd- $\omega$ pairs have also been explored, including its connection to zeroenergy density of states (DOS) peaks [26-31].

The Cooper pair symmetry follows the $\mathcal{S P O T}=-1$ classification in all systems where the constituent electrons have spin-1/2 character. There exist, however, recent reports on superconductivity in materials where the low-energy electrons also carry a finite orbital angular momenta, such that only the total angular momentum is a good quantum number [32,33]. For example, some cubic half-Heusler compounds, with the general form RPtBi or RPdBi where $\mathrm{R}$ is the rare-earth element, host "spin"-3/2 low-energy fermions and have been shown to be superconducting at low temperatures [32]. In these materials the conserved total angular momentum, or effective, spin- $3 / 2$ character is protected by high crystal symmetry and spin-orbit interaction. These findings have drawn a large amount of attention since the superconducting ground state has been found to have several unusual properties, such as a reduced upper critical field [33] and a low-temperature penetration depth [34] very different from the properties of conventional spin-singlet superconductors [35]. Notably, the 
spin-3/2 character of the constituent electrons of the Cooper pairs allows for both exotic spin quintet with $j=2$ and spin septet with $j=3$ pairing [32,33,36-38], in addition to the spin-singlet $(j=0)$ and spin-triplet $(j=1)$ pairing found in traditional spin- $1 / 2$ superconductors, where $j$ is the total angular momentum quantum number of the Cooper pair.

Interestingly, superconductivity in spin-3/2 systems can be associated with a Bogoliubov Fermi surface (BFS); an inflated spheroidal or toroidal topologically protected region in momentum space across which the energy gap is identically zero [39-42]. The reason behind the appearance of a BFS has been described in terms of a pseudomagnetic field arising from exotic pairing states [39]. These exotic pairing states have been predicted in a range of materials: nematic superconductors [43], iron-based superconductors [44,45], and also cubic half-Heusler compounds [32,46-49]. The general prescription for the appearance of BFS is the existence of an internal electronic degree of freedom, in addition to the spin degree of freedom, such as an additional orbital or sublattice degree of freedom, subjected to the symmetries of the system $[40,50,51]$. Higher spin, such as a spin- $3 / 2$ character, of the low-energy bands is perhaps the most explored possibility for that internal degree of freedom to produce a BFS [40].

The BFS manifests itself through a large DOS at zero energy in the superconducting state [41]. At the same time, zero-energy states have also been shown to be one of the possible signatures of odd- $\omega$ pair amplitudes in many systems $[7,26,52]$. A natural question then arises: is BFS a manifestation of odd- $\omega$ pairing? This question is also closely related to developing a more solid understanding of the exotic pairing present in materials with BFSs. In this work we address these issues by focusing on spin-3/2 superconductors, and particularly cubic half-Heusler compounds, as they present possibilities for both BFS and exotic pairing with higher angular momentum Cooper pairs whose complete symmetry classification is not yet fully developed.

In particular, we first introduce a complete classification for higher-spin superconductors: $\mathcal{J P} \mathcal{T}=-1$, where $\mathcal{J}$ is the exchange operator for the quantum numbers associated with the $z$ component of the total angular momenta of the constituent electrons of the Cooper pairs. We then establish the applicability of this new classification by studying several generic and realistic models describing superconducting halfHeusler compounds and calculating the superconducting pair amplitudes and their symmetries. The $\mathcal{J P} \mathcal{T}=-1$ antisymmetry condition gives rise to a total of 32 different classes of Cooper pair symmetry, and we find that essentially all can exist in the half-Heusler compounds. In particular, we find all types of possible odd- $\omega$ pairs: spin-singlet odd-parity, spintriplet even-parity, spin-quintet odd-parity, and spin-septet even-parity, which in several cases are as large as any even- $\omega$ components. Following this, we derive a general analytical expression for the odd- $\omega$ pair amplitude in spin- $3 / 2$ systems, which we then use to derive a necessary and sufficient condition for finding both odd- $\omega$ pairs and a BFS. Finally, by using a low-energy effective two-band model, we show explicitly that the pseudomagnetic field responsible for the BFS also always induces odd- $\omega$ pairing and vice versa. This establishes a one-to-one correspondence between BFS and odd- $\omega$ pairs in a low-energy model of spin-3/2 superconductors.
The rest of this work is organized as follows. We classify the superconducting pair symmetries in Sec. II and show the appearance of those symmetries in Sec. III by considering a generic Hamiltonian suitable for half-Heusler compounds (Sec. III A), followed by numerical results for the superconducting pair amplitude (Sec. III B). We then find a general analytical expression for odd- $\omega$ pair amplitudes applicable to any spin-3/2 system in Sec. IV and explore the connection of the odd- $\omega$ pair amplitudes to BFSs in Sec. V. Finally, we summarize and conclude our results in Sec. VI.

\section{CLASSIFICATION OF PAIR SYMMETRY IN SPIN-3/2 SYSTEMS}

We begin by developing the classification of the pair symmetry for any superconductor with low-energy electrons of spin-3/2 character. Central in any such discussion is the superconducting pair amplitude, or Cooper pair amplitude, given by the anomalous Green's function as

$\mathcal{F}_{\sigma_{1} a_{1} ; \sigma_{2} a_{2}}\left(\boldsymbol{k}_{1}, \boldsymbol{k}_{2} ; t_{1}, t_{2}\right)=-i\left\langle\mathcal{T}_{t} c_{\sigma_{1} a_{1}}\left(\boldsymbol{k}_{1}, t_{1}\right) c_{\sigma_{2} a_{2}}\left(\boldsymbol{k}_{2}, t_{2}\right)\right\rangle$,

where $\mathcal{T}_{t}$ is the time-ordering operator and $c_{\sigma, a}(\boldsymbol{k}, t)$ is the annihilation operator for an electron in orbital $a$ with spin $\sigma$ and momentum $\boldsymbol{k}$ at time $t$ [53]. Now, for regular spin-1/2 systems, there exists an antisymmetry condition:

$$
\mathcal{S P O T F} \mathcal{F}_{\sigma_{1} a_{1}, \sigma_{2} a_{2}}\left(\boldsymbol{k}_{1}, \boldsymbol{k}_{2} ; t_{1}, t_{2}\right)=-\mathcal{F}_{\sigma_{1} a_{1}, \sigma_{2} a_{2}}\left(\boldsymbol{k}_{1}, \boldsymbol{k}_{2} ; t_{1}, t_{2}\right) \text {, }
$$

imposed by the fermionic nature of the electrons of the Cooper pair where the $\mathcal{S}, \mathcal{P}, \mathcal{O}$, and $\mathcal{T}$ operators are defined as

$$
\begin{aligned}
\mathcal{S} \mathcal{F}_{\sigma_{1} a_{1}, \sigma_{2} a_{2}}\left(\boldsymbol{k}_{1}, \boldsymbol{k}_{2} ; t_{1}, t_{2}\right) & =\mathcal{F}_{\sigma_{2} a_{1}, \sigma_{1} a_{2}}\left(\boldsymbol{k}_{1}, \boldsymbol{k}_{2} ; t_{1}, t_{2}\right) \\
& = \pm \mathcal{F}_{\sigma_{1} a_{1}, \sigma_{2} a_{2}}\left(\boldsymbol{k}_{1}, \boldsymbol{k}_{2} ; t_{1}, t_{2}\right), \\
\mathcal{P} \mathcal{F}_{\sigma_{1} a_{1}, \sigma_{2} a_{2}}\left(\boldsymbol{k}_{1}, \boldsymbol{k}_{2} ; t_{1}, t_{2}\right) & =\mathcal{F}_{\sigma_{1} a_{1}, \sigma_{2} a_{2}}\left(\boldsymbol{k}_{2}, \boldsymbol{k}_{1} ; t_{1}, t_{2}\right) \\
& = \pm \mathcal{F}_{\sigma_{1} a_{1}, \sigma_{2} a_{2}}\left(\boldsymbol{k}_{1}, \boldsymbol{k}_{2} ; t_{1}, t_{2}\right), \\
\mathcal{O} \mathcal{F}_{\sigma_{1} a_{1}, \sigma_{2} a_{2}}\left(\boldsymbol{k}_{1}, \boldsymbol{k}_{2} ; t_{1}, t_{2}\right) & =\mathcal{F}_{\sigma_{1} a_{2}, \sigma_{2} a_{1}}\left(\boldsymbol{k}_{1}, \boldsymbol{k}_{2} ; t_{1}, t_{2}\right) \\
& = \pm \mathcal{F}_{\sigma_{1} a_{1}, \sigma_{2} a_{2}}\left(\boldsymbol{k}_{1}, \boldsymbol{k}_{2} ; t_{1}, t_{2}\right), \\
\mathcal{T} \mathcal{F}_{\sigma_{1} a_{1}, \sigma_{2} a_{2}}\left(\boldsymbol{k}_{1}, \boldsymbol{k}_{2} ; t_{1}, t_{2}\right) & =\mathcal{F}_{\sigma_{1} a_{1}, \sigma_{2} a_{2}}\left(\boldsymbol{k}_{1}, \boldsymbol{k}_{2} ; t_{2}, t_{1}\right) \\
& = \pm \mathcal{F}_{\sigma_{1} a_{1}, \sigma_{2} a_{2}}\left(\boldsymbol{k}_{1}, \boldsymbol{k}_{2} ; t_{1}, t_{2}\right) .
\end{aligned}
$$

The antisymmetry condition of the pair symmetries can in short form be written as $\mathcal{S P O T}=-1$ and applies to any spin-1/2 superconductor, where each electron of the Cooper pair possesses zero orbital angular momentum $\left(l_{i}=0\right)$ and half spin $\left(s_{i}=1 / 2\right)$ quantum numbers, with $i(=1,2)$ being the electron number index. Thus, the total angular momentum for each electron is $j_{i}=l_{i}+s_{i}=1 / 2$. There are two possible spin symmetries for spin- $1 / 2$ systems since $\frac{1}{2} \otimes \frac{1}{2}=0 \oplus 1$ : spin-singlet with total "spin" $j=s=0$ and $m=0$ and spintriplet states with total spin $j=s=1$ and $m=0,1$. Here $m$ is the quantum number for the $z$ component of the total angular momentum, also referred to as secondary total angular momentum quantum number. The Cooper pairs with these spin structures can also be identified according to their spatial parities: explicitly, even-parity ( $s$-wave, $d$-wave, etc.) and odd-parity ( $p$-wave, $f$-wave, etc.) pairs. Given the spin structure and spatial parity, the Cooper pair symmetry can further be classified according to its evenness or oddness under the 
orbital index (if such exists) and then finally with respect to the time/frequency dependence. We note that pair amplitudes that are odd with respect to the change of the time (or equivalently frequency) of the two electrons are identified as odd- $\omega$ pair amplitudes. As an example, all spin-triplet even-parity and all spin-singlet odd-parity states are necessarily odd- $\omega$ states when they are even with respect to exchange of the orbital index [1,11]. Similarly, we can also find other possible odd- $\omega$ pair symmetries when the pair amplitude is odd in orbital index [11].

With the above outline of the pair symmetry classification for spin-1/2 fermions, we now move on to the generalization of the antisymmetry condition for higher spin systems, since the condition Eq. (2) no longer remains applicable when the spin and orbital are strongly coupled to each other. In fact, for such strong spin-orbit coupled systems, both the spin and orbital angular momentum are not good quantum numbers at all. Instead, the total angular momentum of each electron is a good quantum number, and it becomes natural to consider that instead when identifying the superconducting pair symmetry. More precisely, when two electrons of total angular momenta $j_{1}$ and $j_{2}$ pair, the total angular momentum quantum number $j$ for the paired state is constrained by the condition $\left|j_{1}-j_{2}\right| \leqslant j \leqslant\left(j_{1}+j_{2}\right)$. The quantum number associated with the $z$ component of the total angular momentum $m$ follows $-j \leqslant m \leqslant j$. It also satisfies $m=m_{1}+m_{2}$ where $m_{1}$ and $m_{2}$ are the quantum numbers for the $z$ component of the total angular momentum of the two electrons, individually satisfying $-j_{i} \leqslant m_{i} \leqslant j_{i}$. The paired state $|j, m\rangle$ can be expanded in the basis $\left|j_{1}, j_{2} ; m_{1}, m_{2}\right\rangle$ using the completeness and normalization conditions as

$$
\begin{aligned}
|j, m\rangle & =\sum_{m_{1}=-j_{1}}^{j_{1}} \sum_{m_{2}=-j_{2}}^{j_{2}}\left|j_{1}, j_{2} ; m_{1}, m_{2}\right\rangle\left\langle j_{1}, j_{2} ; m_{1}, m_{2} \mid j, m\right\rangle \\
& =\sum_{m_{1}, m_{2}} \underbrace{\left\langle j_{1}, j_{2} ; m_{1}, m_{2} \mid j, m\right\rangle}_{\text {C.G. coefficient }}\left|j_{1}, j_{2} ; m_{1}, m_{2}\right\rangle,
\end{aligned}
$$

where the Clebsch-Gordon (C.G.) coefficients are scalar numbers [54].

In particular, we are here interested in strongly spin-orbit coupled systems where the low-energy band structure has a spin-3/2 character $[33,55]$. When two such electrons with $j_{1}=3 / 2$ and $j_{2}=3 / 2$ couple to form a Cooper pair, the possible states can be found following the product $\frac{3}{2} \otimes \frac{3}{2}=$ $0 \oplus 1 \oplus 2 \oplus 3[32,33,36,37,55,56]$. Thus, for such higher spin system, the pair symmetries are enriched by five spin-quintet $(j=2)$ and seven spin-septet $(j=3)$ states, in addition to extended spin-singlet $(j=0)$ and extended spin-triplet $(j=1)$ states. ${ }^{1}$ Henceforth, we use the short notation $\left|m_{1}, m_{2}\right\rangle$ instead of $\left|j_{1}, j_{2} ; m_{1}, m_{2}\right\rangle$ for brevity since $j_{1}=j_{2}=3 / 2$.

With the concept of the spin structures of the Cooper pairs for spin-3/2 fermions clear, the next natural question is about the overall symmetry of the pairing. We note that, for spin- $1 / 2$

\footnotetext{
${ }^{1}$ One can here raise the question about the possibility of one electron having $j_{1}=1 / 2$ and another having $j_{2}=3 / 2$ character. We avoid considering this case as it is unlikely a Fermi surface includes two electrons of such different spin characters.
}

systems, we have four symmetry operators $\mathcal{S}, \mathcal{P}, \mathcal{O}$, and $\mathcal{T}$ corresponding to the spin quantum number, spatial parity, orbital, and time for the Cooper pairs. However, due to the strong spin-orbit coupling in the spin-3/2 systems, we can now not separately identify the spin and orbital quantum numbers. Instead, the total angular momentum and its $z$ component are the only good quantum numbers. Thus, we now rewrite the anomalous Green's function of the Cooper pair of Eq. (1) characterized by quantum numbers for angular momentum, spatial parity, and time as

$$
\mathcal{F}_{j_{1} m_{1}, j_{2} m_{2}}\left(\boldsymbol{k}_{1}, \boldsymbol{k}_{2} ; t_{1}, t_{2}\right)=-i\left\langle\mathcal{T}_{t} c_{j_{1} m_{1}}\left(\boldsymbol{k}_{1}, t_{1}\right) c_{j_{2} m_{2}}\left(\boldsymbol{k}_{2}, t_{2}\right)\right\rangle,
$$

where $c_{j m}(\boldsymbol{k}, t)$ is now the annihilation operator for an electron with the total angular momentum quantum numbers $j, m$ and spatial momentum $\boldsymbol{k}$ at time $t$. Note that each total angular momentum quantum number $j_{i}$ allows various states identified by $m_{i}$ following Eq. (4) and thus we must include the $m_{i}$ index too. We then introduce a symmetry operator for the exchange of the total angular momentum quantum numbers of the two electrons, $\mathcal{J}$, which effectively exchanges only the $z$ components of the total angular momentum quantum number, since here $j_{1}=j_{2}=3 / 2$. Thus we can now have Cooper pairs which are even or odd with respect to the exchange of the quantum numbers $m_{1} \leftrightarrow m_{2}$ as

$$
\begin{aligned}
\mathcal{J F}_{j_{1} m_{1}, j_{2} m_{2}}\left(\boldsymbol{k}_{1}, \boldsymbol{k}_{2} ; t_{1}, t_{2}\right) & =\mathcal{F}_{\frac{3}{2} m_{2}, \frac{3}{2} m_{1}}\left(\boldsymbol{k}_{1}, \boldsymbol{k}_{2} ; t_{1}, t_{2}\right) \\
& = \pm \mathcal{F}_{\frac{3}{2} m_{1}, \frac{3}{2} m_{2}}\left(\boldsymbol{k}_{1}, \boldsymbol{k}_{2} ; t_{1}, t_{2}\right) .
\end{aligned}
$$

On top of this, the pair amplitudes can again be even or odd with respect to the spatial parity $\mathcal{P}$, as well as (relative) time exchange $\mathcal{T}$ operations, exactly similar to spin- $1 / 2$ fermions [see Eq. (3)]. We note that there is now no separate symmetry operation for the orbital index since it is no longer a good quantum number here, but the orbital index is instead effectively included into the $\mathcal{J}$ operation. Finally, all these exchange operations should follow an antisymmetry condition maintaining the fermionic property of the electrons as

$$
\begin{gathered}
\mathcal{J P} \mathcal{T} \mathcal{F}_{\frac{3}{2} m_{1}, \frac{3}{2} m_{2}}\left(\boldsymbol{k}_{1}, \boldsymbol{k}_{2} ; t_{1}, t_{2}\right) \\
=-\mathcal{F}_{\frac{3}{2} m_{1}, \frac{3}{2} m_{2}}\left(\boldsymbol{k}_{1}, \boldsymbol{k}_{2} ; t_{1}, t_{2}\right) .
\end{gathered}
$$

In short this complies with $\mathcal{J P} \mathcal{T}=-1$, which identify the evenness or oddness of all the possible pairings for spin-3/2 systems with respect to the angular momentum $\mathcal{J}$, spatial parity $\mathcal{P}$, and time $\mathcal{T}$ or frequency.

Having derived the $\mathcal{J P} \mathcal{T}=-1$ condition, we next illustrate how to classify the Cooper pair states following this explicit antisymmetry condition. For this, we first explicitly write out the possible states by using Eq. (4), following Ref. [33], and then show their classification in terms of $\mathcal{J P} \mathcal{T}=$ -1 in Table I. Note that, while the pairing state $\left|m_{1}, m_{2}\right\rangle$ may be either a single state or a combination of two states or more, as seen in the third column in Table I, the operation of $\mathcal{J}\left(m_{1} \leftrightarrow m_{2}\right)$ is additive for each state and thus it always gives either an even or odd Cooper pair state with respect to this exchange. Overall, in Table I there are one singlet, three triplet $\left(t_{1}, t_{2}, t_{3}\right)$, five quintet $\left(q_{1}, q_{2}, q_{3}, q_{4}, q_{5}\right)$, and seven septet $\left(s_{1}, s_{2}, s_{3}, s_{4}, s_{5}, s_{6}, s_{7}\right)$ states, such that a 
TABLE I. Complete symmetry classification of Cooper pairs for $j=3 / 2$ fermions following the antisymmetry condition $\mathcal{J P} \mathcal{T}=-1$. Cooper pair configurations in column 3 follow Ref. [33].

\begin{tabular}{|c|c|c|c|c|c|}
\hline Class & Pairing state & $\begin{array}{c}\text { Cooper pair } \\
\left|j_{1}, m_{1} ; j_{2}, m_{2}\right\rangle \equiv\left|m_{1}, m_{2}\right\rangle\end{array}$ & $\begin{array}{l}\text { Angular momentum } \\
\qquad(\mathcal{J})\end{array}$ & $\begin{array}{l}\text { Parity } \\
(\mathcal{P})\end{array}$ & $\begin{array}{l}\text { Freq./Time } \\
\qquad(\mathcal{T})\end{array}$ \\
\hline \multirow[t]{2}{*}{1} & Singlet (sing.) & \multirow[b]{2}{*}{$\frac{1}{2}\left(\left|\frac{3}{2},-\frac{3}{2}\right\rangle-\left|-\frac{3}{2}, \frac{3}{2}\right\rangle-\left|\frac{1}{2},-\frac{1}{2}\right\rangle+\left|-\frac{1}{2}, \frac{1}{2}\right\rangle\right)$} & \multirow[b]{2}{*}{ Odd } & Even & Even \\
\hline & $(j=0 ; m=0)$ & & & Odd & Odd \\
\hline \multirow[t]{2}{*}{2} & Triplet $\left(t_{1}\right)$ & \multirow[b]{2}{*}{$\frac{1}{\sqrt{10}}\left(\sqrt{3}\left|-\frac{3}{2}, \frac{1}{2}\right\rangle-2\left|-\frac{1}{2},-\frac{1}{2}\right\rangle+\sqrt{3}\left|\frac{1}{2},-\frac{3}{2}\right\rangle\right)$} & \multirow[b]{2}{*}{ Even } & Even & Odd \\
\hline & $(j=1 ; m=-1)$ & & & Odd & Even \\
\hline \multirow[t]{2}{*}{3} & Triplet $\left(t_{2}\right)$ & \multirow[b]{2}{*}{$\frac{1}{\sqrt{20}}\left(3\left|\frac{3}{2},-\frac{3}{2}\right\rangle-\left|\frac{1}{2},-\frac{1}{2}\right\rangle-\left|-\frac{1}{2}, \frac{1}{2}\right\rangle+3\left|-\frac{3}{2}, \frac{3}{2}\right|\right)$} & \multirow[b]{2}{*}{ Even } & Even & Odd \\
\hline & $(j=1 ; m=0)$ & & & Odd & Even \\
\hline \multirow[t]{2}{*}{4} & Triplet $\left(t_{3}\right)$ & \multirow{2}{*}{$\frac{1}{\sqrt{10}}\left(\sqrt{3}\left|\frac{3}{2},-\frac{1}{2}\right\rangle-2\left|\frac{1}{2}, \frac{1}{2}\right\rangle+\sqrt{3}\left|-\frac{1}{2}, \frac{3}{2}\right\rangle\right)$} & \multirow[b]{2}{*}{ Even } & Even & Odd \\
\hline & $(j=1 ; m=1)$ & & & Odd & Even \\
\hline \multirow[t]{3}{*}{5} & Quintet $\left(q_{1}\right)$ & \multirow{3}{*}{$\frac{1}{\sqrt{2}}\left(\left|-\frac{3}{2},-\frac{1}{2}\right\rangle-\left|-\frac{1}{2},-\frac{3}{2}\right\rangle\right)$} & \multirow{3}{*}{ Odd } & Even & Even \\
\hline & $(j=2 ; m=-2)$ & & & & \\
\hline & & & & Odd & Odd \\
\hline \multirow[t]{3}{*}{6} & Quintet $\left(q_{2}\right)$ & \multirow{3}{*}{$\frac{1}{\sqrt{2}}\left(\left|-\frac{3}{2}, \frac{1}{2}\right\rangle-\left|\frac{1}{2},-\frac{3}{2}\right|\right)$} & \multirow{3}{*}{ Odd } & Even & Even \\
\hline & $(j=2 ; m=-1)$ & & & & \\
\hline & & & & Odd & Odd \\
\hline \multirow[t]{2}{*}{7} & Quintet $\left(q_{3}\right)$ & \multirow[b]{2}{*}{$\frac{1}{2}\left(\left|\frac{3}{2},-\frac{3}{2}\right\rangle+\left|\frac{1}{2},-\frac{1}{2}\right\rangle-\left|-\frac{1}{2}, \frac{1}{2}\right\rangle-\left|-\frac{3}{2}, \frac{3}{2}\right\rangle\right)$} & \multirow[b]{2}{*}{ Odd } & Even & Even \\
\hline & $(j=2 ; m=0)$ & & & Odd & Odd \\
\hline \multirow[t]{2}{*}{8} & Quintet $\left(q_{4}\right)$ & \multirow[b]{2}{*}{$\frac{1}{\sqrt{2}}\left(\left|\frac{3}{2},-\frac{1}{2}\right\rangle-\left|-\frac{1}{2}, \frac{3}{2}\right|\right)$} & \multirow[b]{2}{*}{ Odd } & Even & Even \\
\hline & $(j=2 ; m=1)$ & & & .Odd & Odd \\
\hline \multirow[t]{3}{*}{9} & Quintet $\left(q_{5}\right)$ & \multirow{3}{*}{$\frac{1}{\sqrt{2}}\left(\left|\frac{3}{2}, \frac{1}{2}\right\rangle-\left|\frac{1}{2}, \frac{3}{2}\right\rangle\right)$} & \multirow{3}{*}{ Odd } & Even & Even \\
\hline & $(j=2 ; m=2)$ & & & & \\
\hline & & & & Odd & Odd \\
\hline \multirow[t]{2}{*}{10} & Septet $\left(s_{1}\right)$ & \multirow[b]{2}{*}{$\left|-\frac{3}{2},-\frac{3}{2}\right\rangle$} & \multirow[b]{2}{*}{ Even } & Even & Odd \\
\hline & $(j=3 ; m=-3)$ & & & Odd & Even \\
\hline 11 & Septet $\left(s_{2}\right)$ & & & Even & Odd \\
\hline & $(j=3 ; m=-2)$ & $\frac{1}{\sqrt{2}}\left(\left|-\frac{3}{2},-\frac{1}{2}\right\rangle+\left|-\frac{1}{2},-\frac{3}{2}\right\rangle\right)$ & Even & Odd & Even \\
\hline 12 & Septet $\left(s_{3}\right)$ & & & Even & Odd \\
\hline & $(j=3 ; m=-1)$ & $\frac{1}{\sqrt{5}}\left(\left|-\frac{3}{2}, \frac{1}{2}\right\rangle+\sqrt{3}\left|-\frac{1}{2},-\frac{1}{2}\right\rangle+\left|\frac{1}{2},-\frac{3}{2}\right\rangle\right)$ & Even & O & \\
\hline & & & & Odd & Even \\
\hline 13 & Septet $\left(s_{4}\right)$ & & & Even & Odd \\
\hline & $(j=3 ; m=0)$ & $\frac{1}{\sqrt{20}}\left(\left|\frac{3}{2},-\frac{3}{2}\right\rangle+3\left|\frac{1}{2},-\frac{1}{2}\right\rangle+3\left|-\frac{1}{2}, \frac{1}{2}\right\rangle+\left|-\frac{3}{2}, \frac{3}{2}\right\rangle\right)$ & Even & Odd & Even \\
\hline 14 & Septet $\left(s_{5}\right)$ & & & Even & Odd \\
\hline & $(j=3 ; m=1)$ & $\frac{1}{\sqrt{5}}\left(\left|\frac{3}{2},-\frac{1}{2}\right\rangle+\sqrt{3}\left|\frac{1}{2}, \frac{1}{2}\right\rangle+\left|-\frac{1}{2}, \frac{3}{2}\right\rangle\right)$ & Even & Odd & Even \\
\hline
\end{tabular}


TABLE I. (Continued.)

\begin{tabular}{|c|c|c|c|c|c|}
\hline Class & Pairing state & $\begin{array}{c}\text { Cooper pair } \\
\left|j_{1}, m_{1} ; j_{2}, m_{2}\right\rangle \equiv\left|m_{1}, m_{2}\right\rangle\end{array}$ & $\begin{array}{l}\text { Angular momentum } \\
\qquad(\mathcal{J})\end{array}$ & $\begin{array}{l}\text { Parity } \\
(\mathcal{P})\end{array}$ & $\begin{array}{l}\text { Freq./Time } \\
\qquad(\mathcal{T})\end{array}$ \\
\hline \multirow[t]{3}{*}{15} & Septet $\left(s_{6}\right)$ & \multirow{3}{*}{$\frac{1}{\sqrt{2}}\left(\left|\frac{3}{2}, \frac{1}{2}\right\rangle+\left|\frac{1}{2}, \frac{3}{2}\right\rangle\right)$} & \multirow{3}{*}{ Even } & Even & Odd \\
\hline & $(j=3 ; m=2)$ & & & & \\
\hline & & & & Odd & Even \\
\hline \multirow[t]{3}{*}{16} & Septet $\left(s_{7}\right)$ & \multirow{3}{*}{$\left|\frac{3}{2}, \frac{3}{2}\right\rangle$} & \multirow{3}{*}{ Even } & Even & Odd \\
\hline & \multirow[t]{2}{*}{$(j=3 ; m=3)$} & & & 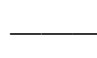 & \\
\hline & & & & Odd & Even \\
\hline
\end{tabular}

total of 16 possible spin structures exist for spin-3/2 fermions. Considering also spatial parity and time, each of these 16 states can be further characterized either by being odd or even under parity and time, resulting in a maximum of 32 possible states for spin-3/2 fermions, as shown in Table I.

We next explicitly illustrate some of the symmetries of Table I. The spin-singlet state for spin-3/2 fermions can be thought of as a combination of two separate states formed by the first two and last two parts of the Cooper pair as $\frac{1}{2}\left(\left|\frac{3}{2},-\frac{3}{2}\right\rangle-\left|-\frac{3}{2}, \frac{3}{2}\right\rangle\right)$ and $\frac{1}{2}\left(-\left|\frac{1}{2},-\frac{1}{2}\right\rangle+\left|-\frac{1}{2}, \frac{1}{2}\right\rangle\right)$, also called an extended singlet state [33]. When the $\mathcal{J}$ operator operates on each of these combined states, it exchanges the $m_{i}$ quantum numbers of the two electrons of each individual parts following Eq. (6), i.e., $3 / 2 \leftrightarrow-3 / 2$ for the first state and $1 / 2 \leftrightarrow-1 / 2$ for the last state and both combined states are thus odd with respect to this exchange operation. Since the operation of $\mathcal{J}$ is additive, the whole singlet state is also odd. Now, this odd state can further be both even and odd with respect to the spatial parity $\mathcal{P}$ and in time $\mathcal{T}$ or frequency. Following $\mathcal{J P} \mathcal{T}=-1$, the singlet state has to be either even-parity even- $\omega$ or odd-parity odd- $\omega$, as also seen in Table I.

Next we consider one of the triplet states, triplet $t_{1}$, in Table I, which consists of two parts: $\frac{\sqrt{3}}{10}\left(\left|-\frac{3}{2}, \frac{1}{2}\right\rangle+\left|\frac{1}{2},-\frac{3}{2}\right\rangle\right)$ and $-\frac{2}{10}\left|-\frac{1}{2},-\frac{1}{2}\right\rangle$. When $\mathcal{J}$ operates on these combined states, the corresponding exchange operations are $-3 / 2 \leftrightarrow$ $1 / 2$ and $-1 / 2 \leftrightarrow-1 / 2$, in the first and last states, respectively, and these exchange operations show that the pair amplitude for this triplet state $t_{1}$ is even with respect to $\mathcal{J}$. Note that, in the latter case, the state is self-exchanging under the operation $-1 / 2 \leftrightarrow-1 / 2$, since both the electrons are characterized by the same $z$ components of the total angular momentum quantum numbers. The other two spin triplets, triplets $t_{2}$ and $t_{3}$, are also even states under the exchange operation $\mathcal{J}$ in the same way. Then, according to the $\mathcal{J P} \mathcal{T}=-1$ antisymmetry condition, all the spin-triplet states can be either even-parity odd- $\omega$ or odd-parity even- $\omega$ states. Similarly, we classify all the spin-quintet $\left(q_{j}\right)$ and spin-septet $\left(s_{j}\right)$ states. All five spin-quintet states are odd in $\mathcal{J}$. These spin-quintet states can thus further be categorized as either even-parity even- $\omega$ or odd-parity odd- $\omega$, similar to the spin-singlet state. Similarly, all seven spin septets can be either even-parity odd- $\omega$ or oddparity even- $\omega$. Note that septet $s_{1}$ and $s_{7}$ states are states that change into themselves under the exchange operation by $\mathcal{J}$, thus always resulting in $\mathcal{J}=+1$. All of these symmetries are explicitly written out in Table I.

\section{EXAMPLES OF PAIR SYMMETRIES FOR SPIN-3/2 FERMIONS}

With the classification for spin-3/2 fermion systems in the previous section, we now show the existence of all those pair symmetries by considering a generic normal-state Hamiltonian suitable for half-Heusler compounds which are known to both host low-energy spin-3/2 fermions due to the strong spin-orbit coupling and be superconducting [32]. We here consider several different superconducting pair potentials following the superconducting behaviors of these materials $[32,40]$.

\section{A. Model for superconducting spin-3/2 fermion systems}

We start with the Bogoliubov-de Gennes (BdG) Hamiltonian given by

$$
\check{H}=\frac{1}{2} \sum_{k} \Psi_{k}^{\dagger} \check{\mathcal{H}}_{k} \Psi_{k},
$$

where $\Psi_{k}=\left(c_{k}, c_{-k}^{\dagger}\right)^{T}$ is the Nambu spinor with $c_{k}$ being a four-component spinor encoding the internal degrees of freedom for spin-3/2 fermions, $c_{k}=\left(c_{k, 3 / 2}, c_{k, 1 / 2}, c_{k,-1 / 2}, c_{k,-3 / 2}\right)^{T}, \quad$ and $\quad k=\left\{k_{x}, k_{y}, k_{z}\right\}$. Here

$$
\check{\mathcal{H}}_{k}=\left(\begin{array}{cc}
\hat{H}_{0}(\boldsymbol{k}) & \hat{\Delta}(\boldsymbol{k}) \\
\hat{\Delta}^{\dagger}(\boldsymbol{k}) & -\hat{H}_{0}^{T}(-\boldsymbol{k})
\end{array}\right),
$$

where we label $8 \times 8$ and $4 \times 4$ matrices operator by $\therefore$ and $\hat{\ldots}$, respectively. We here consider cubic materials where the low-energy bands of a strongly spin-orbit coupled system can be described by a generic $\boldsymbol{k} \cdot \boldsymbol{p}$ model Hamiltonian appropriate for half-Heusler materials, but neglecting higher order terms as they do not affect our conclusions qualitatively [39,57]. In particular, we write the normal part of the BdG Hamiltonian [39] as

$$
\begin{aligned}
\hat{H}_{0}(\boldsymbol{k})= & \alpha k^{2} \hat{I}+\beta \sum_{\nu} k_{v}^{2} \hat{J}_{v}^{2}+\gamma \sum_{\nu \neq v^{\prime}} k_{\nu} k_{\nu^{\prime}} \hat{J}_{v} \hat{J}_{\nu^{\prime}} \\
& +\delta \sum_{\nu} k_{v}\left(\hat{J}_{v+1} \hat{J}_{v} \hat{J}_{v+1}-\hat{J}_{v+2} \hat{J}_{v} \hat{J}_{v+2}\right)-\mu,
\end{aligned}
$$

where $\hat{I}$ is the identity operator and $\hat{J}_{v}$, with $v \in\{x, y, z\}$, are the $4 \times 4$ matrices for spin- $3 / 2$ fermions (see the Appendix). Here $v+1$ and $v+2$ follow the cyclic shift operations on $\{x, y, z\}$. For each momentum $\boldsymbol{k}, \hat{H}_{0}(\boldsymbol{k})$ thus has four electronic degrees of freedom, arising from the four channels 
with $m= \pm 3 / 2$ and $\pm 1 / 2$. Here, $\alpha, \beta, \gamma$, and $\delta$ are all real constants and material dependent. The kinetic energy part involves $\alpha$, while $\beta$ and $\gamma$ characterize the symmetric spinorbit coupling strength. Moreover, $\delta$ is proportional to the asymmetric part of the spin-orbit interaction and it breaks the inversion symmetry of the normal-state Hamiltonian and (as we will see) plays a vital role for generating an odd-parity pair amplitude. We here choose the parameter values $a=1, \alpha=$ $20.5(a / \pi)^{2} \mathrm{eV}, \beta=-18.5(a / \pi)^{2} \mathrm{eV}, \gamma=-12.7(a / \pi)^{2} \mathrm{eV}$, and $\delta=0.06(a / \pi) \mathrm{eV}$ as they nicely capture all the essential features of the band structures for YPtBi, which is one of the superconducting materials showing spin-3/2 character [32]. We further fix the chemical potential $\mu=50 \mathrm{meV}$ (intrinsic doping) as it encodes the spin-orbit split holelike Fermi surface consistent with literature [33]. We note that our results of the pair symmetry classification are robust to changes in the parameter values and the behavior of the pair amplitudes also remain qualitatively similar.

Having the Hamiltonian in Eq. (9) we define the Green's function as

$$
\breve{G}=(i \omega-\check{H})^{-1}=\left(\begin{array}{ll}
\hat{\mathcal{G}} & \hat{\mathcal{F}} \\
\hat{\hat{\mathcal{F}}} & \hat{\mathcal{G}}
\end{array}\right),
$$

where $\hat{\mathcal{G}}(\hat{\overline{\mathcal{G}}})$ and $\hat{\mathcal{F}}(\hat{\overline{\mathcal{F}}})$ are the $4 \times 4$ normal and anomalous Green's functions in particle (hole) space, respectively. For superconductivity we are particularly interested in $\mathcal{F}$, where each element is characterized by $j_{1}, m_{1} ; j_{2}, m_{2}$ as explicitly written in Eq. (5).

\section{B. Numerical results for pair amplitudes}

To establish the existence of the symmetry classes in Table I, we consider different superconducting pair potentials of $\hat{\Delta}(\boldsymbol{k})$ for the BdG Hamiltonian Eq. (9) and calculate the anomalous Green's function using Eq. (11). We start with the simplest even-parity $s$-wave pair potential and then also consider odd-parity $p$-wave, followed by chiral even-parity ( $d$-wave) pair potentials since all these have been suggested to describe spin- $3 / 2$ superconductors in the current literature $[32,33,40,58]$.

\section{Even-parity (s-wave) pair potential}

We start by considering a momentum independent $s$-wave superconducting pair potential as it is the simplest possible form. For spin-3/2 systems it takes the form [40]

$$
\hat{\Delta}(\boldsymbol{k})=\Delta_{S} U_{T}
$$

with the $s$-wave gap $\Delta_{s}$ being a real constant and the unitary operator $U_{T}$ defined in the Appendix.

We calculate the real and imaginary parts of the anomalous Green's function following Eq. (11) in order to extract the pair amplitudes and present them in Fig. 1. To avoid any mixing of spatial parity, we always symmetrize $\hat{\mathcal{F}}$ by breaking it up into its even- and odd-parity parts: $\mathcal{F}^{\mathrm{Pe}}=\mathcal{F}(\boldsymbol{k})+\mathcal{F}(-\boldsymbol{k})$ and $\mathcal{F}^{\text {Po }}=\mathcal{F}(\boldsymbol{k})-\mathcal{F}(-\boldsymbol{k})$. We here take the summation over positive $\boldsymbol{k}$ from zero to a cut-off value of the momentum $k_{c}=$ $\pi / 2$, but our results do not qualitatively depend on the cut-off. From both the real and imaginary parts of the pair amplitude in Figs. 1(a) and 1(b) we see that the spin-singlet even-parity pair

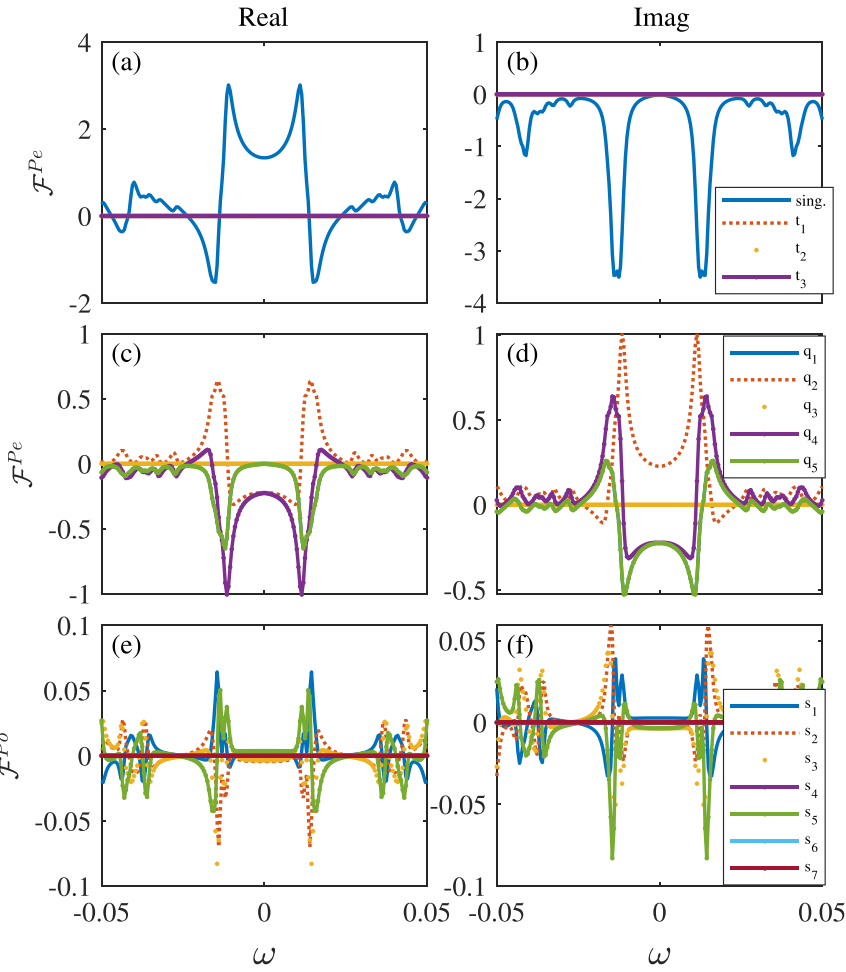

FIG. 1. Real (a),(c),(e) and imaginary (b),(d),(f) parts of $\mathcal{F}^{\mathrm{Pe}}$ (a)-(d) and $\mathcal{F}^{\mathrm{Po}}$ (e),(f) as a function of frequency $\omega$ considering the pair potential of Eq. (12) with $\Delta_{s}=0.01 \mathrm{eV}$. The rest of the pair amplitudes are zero.

amplitude has an even frequency dependency (even- $\omega$ ) with the higher values of the pair amplitude appearing for lower values of $\omega$. On the other hand, all the spin-triplet even-parity pair amplitudes are zero. For all the remaining figures in this section, if a pair amplitude is not plotted, it is because it is identically zero.

Similar to the spin-singlet pair amplitude, all five spinquintet pair amplitudes in Figs. 1(c) and 1(d) are also even- $\omega$, since they are even in parity. Note that one of the spin quintets, quintet $q_{3}$, is zero, while the $q_{1}$ and $q_{5}$ pair amplitudes are equal to each other. Similar to the spin-singlet amplitudes, three of the spin-quintet pair amplitudes also show a large peak for low $\omega$. Finally, moving on to the spin-septet part in Fig. 1, we find that all the spin-septet even-parity pair amplitudes are identically zero. Instead, it is the spin-septet odd-parity pair amplitudes that exist for an $s$-wave pair potential. Note that three of the spin septets, $s_{4}, s_{6}, s_{7}$, are here identically zero. However, a basis rotation will interchange which spin septets are zero, and we can in this way confirm that all spin-septet amplitudes are odd parity and even- $\omega$. A similar procedure and result are also present for the zero spin-quintet amplitude discussed above.

Based on the results in Fig. 1 we conclude that the spinsinglet even-parity pair amplitude is even- $\omega$ and very similar to what happens in spin-1/2 systems [1]. Additionally, we find spin-quintet even-parity even- $\omega$ pair amplitudes and spinseptet odd-parity even- $\omega$ pair amplitudes, which are both unique for higher spin systems. The nonzero spin-quintet and spin-septet pair amplitudes are similar in magnitude but 

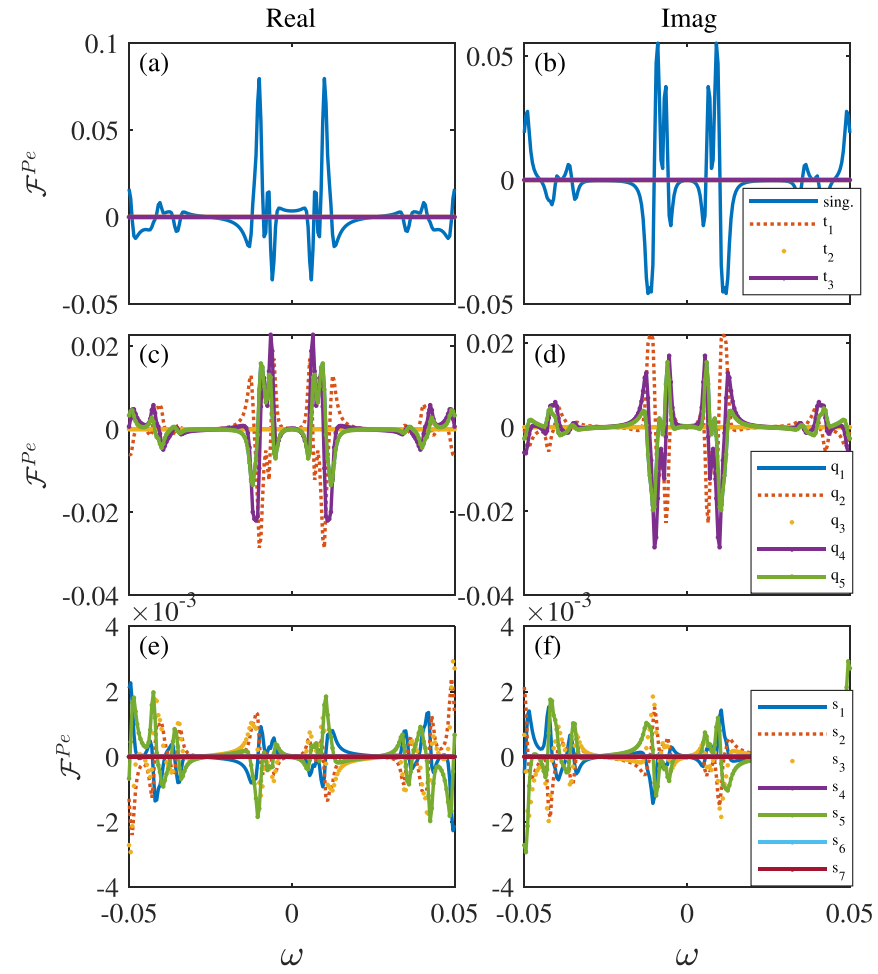

FIG. 2. Real (a),(c),(e) and imaginary (b),(d),(f) parts of $\mathcal{F}^{\mathrm{Pe}}$ as a function of frequency $\omega$ using the pair potential of Eq. (13) with $\Delta_{p}=0.01 \mathrm{eV}$.

smaller than that of spin-singlet pair amplitude. All these pair symmetries found for the $s$-wave pair potential match with the symmetries shown in Table I, explicitly, the even-parity parts of classes 1, 5-6, 8-9, and odd-parity parts of classes 10-12, 14. Notably, there is no odd- $\omega$ pair amplitude (irrespective of parity) present for the $s$-wave pair potential. We explain this absence in Sec. IV.

\section{Odd-parity (p-wave) pair potential}

Next, we move on to the scenario of the odd-parity ( $p$ wave) spin-septet pair potential for spin-3/2 superconductors proposed in Refs. [32,33]. Hence, we use

$$
\hat{\Delta}(\boldsymbol{k})=\Delta_{p}\left(\begin{array}{cccc}
\frac{3}{4} k_{-} & \frac{\sqrt{3}}{2} k_{z} & \frac{\sqrt{3}}{4} k_{+} & 0 \\
\frac{\sqrt{3}}{2} k_{z} & \frac{3}{4} k_{+} & 0 & -\frac{\sqrt{3}}{4} k_{-} \\
\frac{\sqrt{3}}{4} k_{+} & 0 & -\frac{3}{4} k_{-} & \frac{\sqrt{3}}{2} k_{z} \\
0 & -\frac{\sqrt{3}}{4} k_{-} & \frac{\sqrt{3}}{2} k_{z} & -\frac{3}{4} k_{+}
\end{array}\right),
$$

where $k_{ \pm}=k_{x} \pm i k_{y}$ and the $p$-wave triplet gap $\Delta_{p}$ is a real constant. We again calculate the pair amplitude using the anomalous Green's function, Eq. (11). We show the real and imaginary parts of the even- and odd-parity pair amplitudes, i.e., $\hat{\mathcal{F}}^{\mathrm{Pe}}$ and $\hat{\mathcal{F}}^{\mathrm{Po}}$, for the $p$-wave pair potential in Figs. 2 and 3 , respectively.

From Figs. 2(a) and 2(b) we observe that the spin-singlet even-parity pair amplitude is even- $\omega$ and has large amplitude for low values of $\omega$, while the spin-triplet even-parity pair amplitudes are zero. Four of the spin-quintet pair amplitudes $\left(q_{1}, q_{2}, q_{4}\right.$, and $\left.q_{5}\right)$ are also clearly nonzero, with $q_{1}=q_{5}$, and

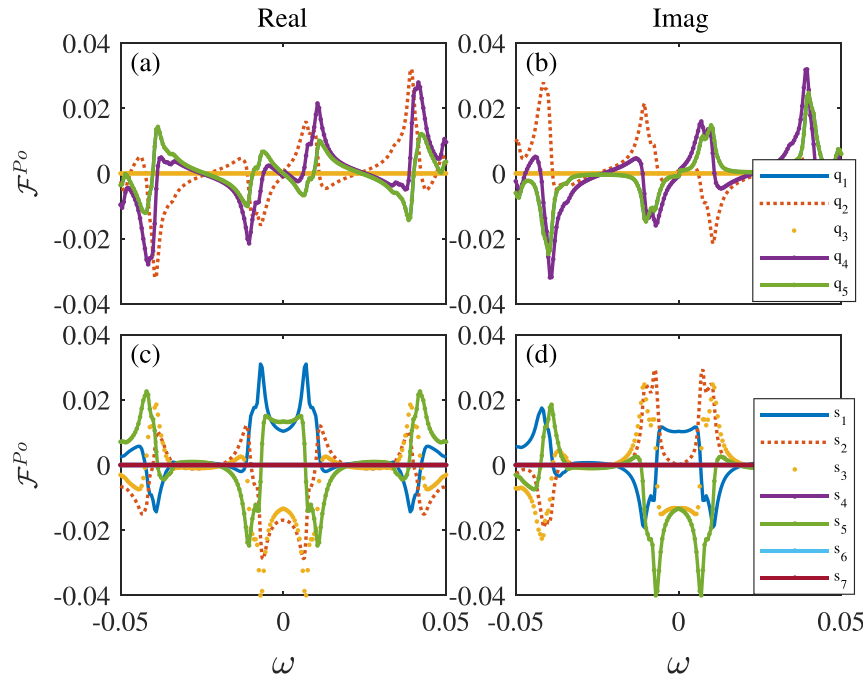

FIG. 3. Real (a),(c) and imaginary (b),(d) parts of $\mathcal{F}^{\mathrm{Po}}$ as a function of frequency $\omega$. The rest of the odd-parity pair amplitudes are zero. The parameter values are the same as in Fig. 2.

all being even- $\omega$ since they are also even parity, as shown in Figs. 2(c) and 2(d). Although the $q_{3}$ pair amplitude is zero in this particular basis, it can be shown to have the same symmetry as the other $q$ pair amplitudes by rotating the basis such that it becomes nonzero. This behavior is the same as what we find for the $s$-wave pair potential in Fig. 1. We also find that four $\left(s_{1}, s_{2}, s_{3}\right.$, and $\left.s_{5}\right)$ out of the seven spin-septet even-parity pair amplitudes are finite, but they all have a small magnitude, as shown in Figs. 2(e) and 2(f). Interestingly, all these spin-septet pair amplitudes are odd- $\omega$, as clearly seen in Figs. 2(e) and 2(f), which are different from all the pair amplitudes discussed so far.

Moving on to the odd-parity $\mathcal{F}^{\text {Po }}$, we find that all the spin-singlet and spin-triplet odd-parity pair amplitudes are zero. However, in Figs. 3(a) and 3(b) we show that the four spin-quintet odd-parity pair amplitudes $\left(q_{1}, q_{2}, q_{4}\right.$, and $q_{5}$, with $\left.q_{1}=q_{5}\right)$ are finite. They are all odd- $\omega$, unlike the spin quintets even parity in Fig. 2. These spin-quintet amplitudes are also all comparable in magnitude with the heights of the peaks of the pair amplitudes gradually increasing with $\omega$ (including finite outside of the plotting window). Here, the $q_{3}$ pair amplitude is zero, but it can be shown that it follows the same symmetry by rotating the basis. Finally, four of the spin-septet odd-parity pair amplitudes, $\left(s_{1}, s_{2}, s_{3}\right.$, and $\left.s_{5}\right)$, are finite and even- $\omega$, as presented in Figs. 3(c) and 3(d) and following the pair amplitude symmetry of Eq. (13). Other septet amplitudes are zero in our choice of basis, but a rotation of the basis can give rise to nonzero values for the remaining septets $\left(s_{4}, s_{6}\right.$, and $\left.s_{7}\right)$ too, which then all follow the same symmetry classification. Notably, all the nonzero spin-quintet and spin-septet odd-parity pair amplitudes are comparable in magnitude and their symmetries follow the classification shown in Table I.

On the whole, in addition to the pair amplitudes found for the $s$-wave pair potential, we here for $p$-wave pair potential find spin-triplet and spin-septet even-parity odd- $\omega$ pair amplitudes as shown in Figs. 2 and 3. These pair amplitudes are in 

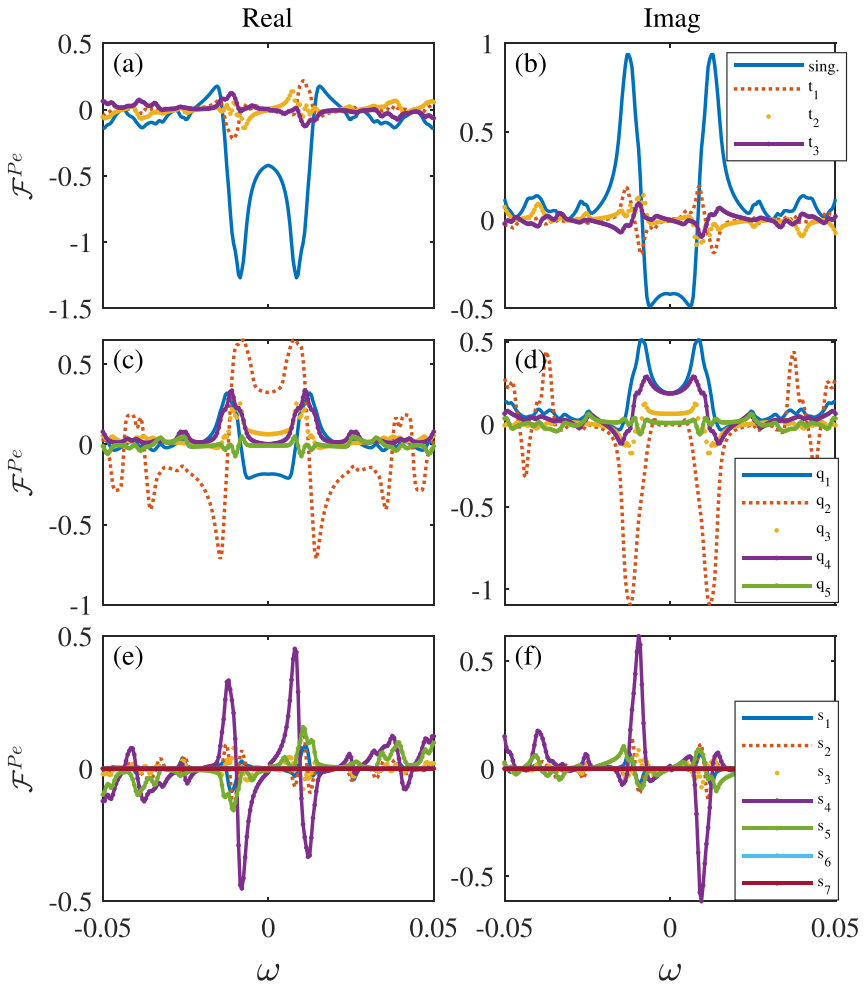

FIG. 4. Real (a),(c),(e) and imaginary (b),(d),(f) parts of $\mathcal{F}^{\mathrm{Pe}}$ as a function of frequency $\omega$ using the pair potential of Eq. (14) with $\Delta_{0}=\Delta_{1}=0.01$.

agreement with the classification shown in Table I, even-parity parts of classes $1,5,6,8-12,14$ and odd-parity parts of classes $5,6,8-12,14$.

\section{Chiral even-parity (d-wave) pair potential}

Finally, we consider the chiral even-parity superconducting pair potential proposed in $[39,59]$,

$$
\hat{\Delta}(\boldsymbol{k})=\Delta_{1} \psi_{k} \eta_{s}+\Delta_{0}\left(\eta_{x z}+i \eta_{y z}\right),
$$

where

$$
\begin{aligned}
& \eta_{y z}=\frac{1}{\sqrt{3}}\left(\hat{J}_{y} \hat{J}_{z}+\hat{J}_{z} \hat{J}_{y}\right) U_{T}, \\
& \eta_{x z}=\frac{1}{\sqrt{3}}\left(\hat{J}_{z} \hat{J}_{x}+\hat{J}_{x} \hat{J}_{z}\right) U_{T}
\end{aligned}
$$

with $\Delta_{1}$ and $\Delta_{0}$ being real constants. This pair potential breaks time-reversal symmetry but retains inversion symmetry. Here, $\eta_{s}$ is a spin-singlet state with a form factor $\psi_{k}$ that is completely isotropic in the pairing channel, i.e., no mixing between the $m=1 / 2$ and $m=3 / 2$ channels. The form factor $\psi_{k}$ breaks time-reversal symmetry but preserves inversion symmetry and is usually taken as $k_{z}\left(k_{x}+i k_{y}\right)$. The gap matrix $\left(\eta_{x z}+i \eta_{y z}\right)$ is chiral and transforms under rotation similarly to the spherical harmonics $Y_{2,1}(k)$ [39]. Instead of point or line nodes as is found generally for a $d$-wave pair potential, this pair potential shows an inflated line node, i.e., a BFS. In fact, it is the presence of a finite $\Delta_{0}$ that is responsible for the appearance of the BFS, whereas in the absence of $\Delta_{0}$, there is only a line node in the $k_{z}=0$ plane and two
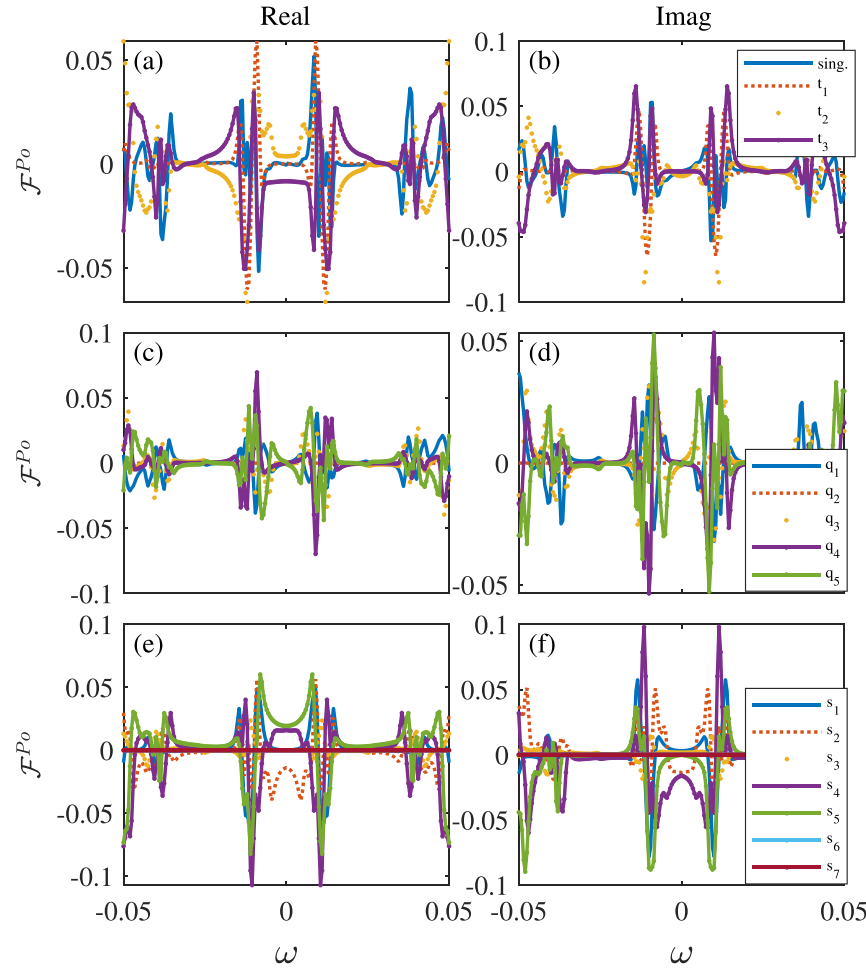

FIG. 5. Real (a),(c),(e) and imaginary (b),(d),(f) parts of $\mathcal{F}^{\text {Po }}$ as a function of frequency $\omega$. The parameter values are the same as in Fig. 4.

point nodes on the $k_{z}$ axis $\left(k_{x}=k_{y}=0\right)$ [39]. Equation (14) is the most considered pair potential in the literature to explain the appearance of BFS in spin-3/2 systems. Thus using this pair potential allows us not just another opportunity to find different pair amplitudes in Table I, but also to investigate a possible relation between the appearance of BFS and odd-frequency pairing. To capture the general behavior of the chiral even-parity pair potential in Eq. (14), we set both $\Delta_{0}$ and $\Delta_{1}$ to nonzero values. We then calculate both the even- and odd-parity spatial parts of $\hat{\mathcal{F}}, \mathcal{F}^{\mathrm{Pe}}$, and $\mathcal{F}^{\text {Po }}$ to find the pair amplitudes and show these in Figs. 4 and 5, respectively. In Figs. 4(a) and 4(b), we see that the spin-singlet even-parity pair amplitude is finite and even- $\omega$. It is similar to the spin-singlet pair amplitude found for the $s$-wave pair potential in Sec. III B 1. However, unlike for the $s$-wave pair potential, here all the spin-triplet $\left(t_{1}, t_{2}\right.$, and $\left.t_{3}\right)$ even-parity pair amplitudes are also finite. Interestingly, these spin-triplet pair amplitudes are odd- $\omega$, following the classification of Table I. Moreover, Figs. 4(c) and 4(d) confirm the even-parity even- $\omega$ behaviors of all the spin-quintet pair amplitudes, in agreement with Table I. Some of the spin-quintet pair amplitudes are already found for $s$-wave pair potentials. We further find that the spin-septet even-parity odd- $\omega$ pair amplitudes are finite and even similar in magnitude to that of the spin-singlet and spin-triplet even- $\omega$ pair amplitudes (except $s_{6}$ and $s_{7}$ ), despite their odd- $\omega$ nature. In particular, we here note that the odd- $\omega$ pair amplitudes for the $p$-wave pair potential in Fig. 2 are two orders of magnitude smaller than what we find here.

We next plot the nonzero odd-parity $\mathcal{F}$ in Fig. 5. From both the real and imaginary parts of Figs. 5(a) and 5(b), we 
see that the spin-singlet pair amplitude is now odd- $\omega$ and all three spin-triplet pair amplitudes, which are even- $\omega$, are finite. This is essentially different from what we find for $s$ - and $p$-wave pair potentials. Moreover, the spin-quintet odd-parity pair amplitudes are odd- $\omega$, while the spin-septet odd-parity pair amplitudes are even- $\omega$. For this chiral pair potential, all the pair amplitudes are comparable in magnitude and mostly higher amplitudes are found for the low values of $\omega$. Thus, all the pair symmetries revealed for the chiral pair potential in Eq. (14) are consistent with Table I, and particularly, we find finite components in almost all classes 1-14 of Table I, with only occasional exceptions where some individual $m$ component is zero.

Having extracted general results for the $d$-wave chiral pair potential in Eq. (14), we note that when $\Delta_{0}=0$, the evenparity spin-triplet and spin-septet pair amplitudes disappear, as well as the odd-parity spin-singlet and spin-quintet pair amplitudes. As a direct consequence, the $\Delta_{0}$ pairing term is responsible for generating all the odd- $\omega$ pair amplitudes. As previously established, a finite $\Delta_{0}$ is also responsible for the appearance of the BFS for this pair potential [39]. This establishes numerically a direct relationship between odd$\omega$ pair amplitudes and BFS for the chiral even-parity pair potential in Eq. (14). Moreover, in the normal-state Hamiltonian, Eq. (10), the spin-orbit inversion symmetry breaking term $\delta$ is the only odd-parity term. Based on symmetry arguments and also confirmed by our numerics, there are no odd-parity pair amplitudes in the absence of this $\delta$ term whenever the superconducting pair potential is even parity, such as for the chiral $d$-wave or the $s$-wave potentials discussed here.

On the whole by studying three different and realistic pair potentials, we show that all different types of the pair symmetries, (1) odd-angular momentum even-parity even- $\omega$, (2) odd-angular momentum odd-parity odd- $\omega$, (3) evenangular momentum even-parity odd- $\omega$, and (4) even-angular momentum odd-parity even- $\omega$ symmetry as classified in Table I, appear for a generic Hamiltonian describing halfHeusler materials. While the classification in Table I itself is universal, independent of the pair potentials and the other parameter values, our three examples show that all these four classes can easily appear in half-Heusler materials. This symmetry classification for spin-3/2 superconductors, and particularly the identification of odd- $\omega$ pair amplitudes, is one of the main results of this work. In fact, through our examples we find all the possible odd- $\omega$ pair amplitudes for spin-3/2 superconductors: (i) spin-singlet odd-parity, (ii) spin-triplet even-parity, (iii) spin-quintet odd-parity, and (iv) spin-septet even-parity pair amplitudes. Among them, we find that the spin-septet even-parity odd- $\omega s_{4}$ pair amplitude for chiral $d$-wave pair potential is largest in magnitude. All the other spin-septet even-parity odd- $\omega$ pair amplitudes and spin-quintet odd-parity odd- $\omega$ pair amplitudes found for the $d$-wave pair potential are also large and comparable in magnitude to all even- $\omega$ pair amplitudes in the same system. In addition to that, also the spin-quintet odd-parity odd- $\omega$ pair amplitudes found for the $p$-wave pair potential are relatively large, only dominated by the spin-singlet even-parity even- $\omega$ pair amplitude.

\section{GENERAL ANALYTICAL EXPRESSION FOR ODD-FREQUENCY PAIR AMPLITUDE}

Having numerically found odd- $\omega$ pair amplitudes with all possible spin structures for spin-3/2 systems, we next derive a general analytical expression for the odd- $\omega$ pair amplitude in these systems. For that, we proceed by rewriting Eq. (11) using Eq. (9) as

$$
\left(\begin{array}{cc}
\hat{\mathcal{G}} & \hat{\mathcal{F}} \\
\hat{\hat{\mathcal{F}}} & \hat{\mathcal{G}}
\end{array}\right)=\left(\begin{array}{cc}
i \omega \hat{\mathcal{I}}-\hat{H}_{0}(\boldsymbol{k}) & -\hat{\Delta}(\boldsymbol{k}) \\
-\hat{\Delta}(\boldsymbol{k}) & i \omega \hat{\mathcal{I}}+\hat{H}_{0}^{T}(-\boldsymbol{k})
\end{array}\right)^{-1} .
$$

We can then write the anomalous Green's function as [12]

$$
\hat{\mathcal{F}}=-\left[\left(i \omega \hat{\mathcal{I}}+\hat{H}_{0}(\boldsymbol{k})\right) \hat{\Delta}(\boldsymbol{k})^{-1}\left(i \omega-\hat{H}_{0}(\boldsymbol{k})\right)-\hat{\Delta}(\boldsymbol{k})\right]^{-1} \text {. }
$$

The pair amplitude can finally be rewritten as

$$
\begin{aligned}
\hat{\mathcal{F}}= & -\left[\left(\hat{\Delta}(\boldsymbol{k})-\hat{\Delta}(\boldsymbol{k})^{-1}\left(\omega^{2}+\hat{H}_{0}(\boldsymbol{k})^{2}\right)\right.\right. \\
& \left.\left.-\hat{\gamma}^{\prime} \hat{H}_{0}(\boldsymbol{k})\right)-i \omega \hat{\gamma}^{\prime}\right] \hat{\mathcal{D}}^{-1},
\end{aligned}
$$

where $\hat{\mathcal{D}}=\left[\left(\hat{\Delta}(\boldsymbol{k})-\hat{\Delta}(\boldsymbol{k})^{-1}\left(\omega^{2}+\hat{H}_{0}(\boldsymbol{k})^{2}\right)-\hat{\gamma}^{\prime} \hat{H}_{0}(\boldsymbol{k})\right)^{2}+\right.$ $\left.\omega^{2} \hat{\gamma}^{\prime 2}\right]$ and $\hat{\gamma}^{\prime}=\left[\hat{H}_{0}(\boldsymbol{k}), \hat{\Delta}(\boldsymbol{k})\right]_{-}$, where the subscript "-, denotes the commutation relation. The denominator $\hat{\mathcal{D}}$, being independent of odd powers of $\omega$, is an even function of $\omega$. Thus the odd- $\omega$ pair amplitude can be easily identified as

$$
\mathcal{F}^{T o}=i \omega \hat{\gamma}^{\prime} \hat{\mathcal{D}}^{-1} \text {. }
$$

We note that this general expression for the odd- $\omega$ pair amplitude is valid for any system where the pair potential $\hat{\Delta}(\boldsymbol{k})$ is a matrix and not a scalar quantity. Thus, Eq. (20) holds for any multiband system, including the spin- $3 / 2$ systems studied here. We note that our expression is more general than that of Ref. [12], where a particular form of multiband Hamiltonian was considered. The odd- $\omega$ pair amplitude includes $\hat{\gamma}^{\prime}$, which is the commutator of the normal-state Hamiltonian and the pair potential. A similar commutation relation has also been used earlier in the context of multiorbital superconductivity when discussing superconducting fitness [60,61], and also applied for spin-3/2 systems [62]. To summarize, it is the incompatibility of the diagonal and off-diagonal elements of Eq. (9) that generates odd-frequency pair amplitudes. This incompatibility further indicates that it is necessarily interorbital terms that are responsible for finite odd- $\omega$ pair amplitudes. This can also be readily confirmed in the results for the $s$-wave pair potential in Sec. III B 1. Here, odd- $\omega$ pair amplitudes are completely absent, despite the plethora of different spin channels, as the $s$-wave pair potential commutes with the normal part of the Hamiltonian.

\section{CONNECTION WITH BOGOLIUBOV FERMI SURFACE}

With the above numerical results and general analytical expression for the odd- $\omega$ pair amplitude, we next look for whether there exists any general connection between the odd$\omega$ pair amplitude and the BFS present in spin-3/2 systems. In particular, the existence of a BFS leads to a finite DOS around zero energy, thus offering an intriguing connection to odd- $\omega$ pair amplitudes, since a finite zero-energy DOS has previously been used as a characteristic feature of the odd- $\omega$ pairing $[7,26,52]$. Moreover, for the chiral $d$-wave pair 
potential in Eq. (14) we have already numerically established a direct relationship between the existence of a BFS and finite odd- $\omega$ pair amplitudes. Taken together, this motivates us to look for analytical connections between BFS and odd- $\omega$ pair amplitudes in spin-3/2 superconductors.

It has recently been shown that the necessary and sufficient condition for the appearance of BFS in spin-3/2 superconductors is that the time-reversal gap product has to be nonzero [40], i.e.,

$$
\hat{\Delta}(\boldsymbol{k}) \hat{\Delta}(\boldsymbol{k})^{\dagger}-\hat{\Delta}_{T}(\boldsymbol{k}) \hat{\Delta}_{T}(\boldsymbol{k})^{\dagger} \neq 0 .
$$

Moreover, as discussed in Sec. IV, for any spin-3/2 superconductor the existence of a finite odd- $\omega$ pair amplitude depends on the noncommuting property of the pair potential $\hat{\Delta}(\boldsymbol{k})$ with the normal-state Hamiltonian $\hat{H}_{0}$ of the superconductor, as expressed by $\hat{\gamma}^{\prime}$ in Eq. (20). Thus we can very generally conclude that odd-frequency pairing and BFS can only both be present for a time-reversal symmetry breaking superconductor pair potential that is incompatible with the normal-state Hamiltonian.

Finally, we perform some further analysis to establish a more explicit and analytical connection between odd- $\omega$ pair amplitude and BFS for spin-3/2 systems, such as the halfHeusler compounds. For this, we only consider the chiral even-parity pair potential of Eq. (14) as this particular choice of pair potential results in a BFS, which is not possible to find using the $s$-wave and $p$-wave pair potentials, and develop an effective low-energy model. To be able to proceed analytically, we first assume that the asymmetric spin-orbit interactions $\gamma, \delta$ in Eq. (10) are zero as they do not play any role in the formation of the BFS. In the absence of these parameters, the energy eigenvalues of the normal-state Hamiltonian of Eq. (10) is doubly degenerate and can be written in the form [32]

$$
\varepsilon_{ \pm}(\boldsymbol{k})=\varepsilon_{0}(\boldsymbol{k}) \pm\left|\boldsymbol{\varepsilon}^{\prime}(\boldsymbol{k})\right|,
$$

where $\varepsilon_{0}(\boldsymbol{k})=\left(\alpha+\frac{5 \beta}{4}\right) \boldsymbol{k}^{2}$ and $\left|\boldsymbol{\varepsilon}^{\prime}(\boldsymbol{k})\right|$ is a compact form of writing the magnitude of a five-dimensional vector, $\left|\boldsymbol{\varepsilon}^{\prime}(\boldsymbol{k})\right|=\sqrt{\sum_{i=1}^{5} \varepsilon_{i}^{2}}$ where $\left\{\varepsilon_{1}, \varepsilon_{2}, \varepsilon_{3}, \varepsilon_{4}, \varepsilon_{5}\right\}=\left\{\sqrt{3} \beta k_{x} k_{y}\right.$,

$$
\hat{\mathcal{H}}_{b-}^{\prime}(\boldsymbol{k})=\left(\begin{array}{cc}
\tilde{\varepsilon}_{\boldsymbol{k}}+h_{z}(\boldsymbol{k}) & \psi_{-}(\boldsymbol{k}) \\
\psi_{-}(\boldsymbol{k}) & -\tilde{\varepsilon}_{\boldsymbol{k}}+h_{z}(\boldsymbol{k}) \\
0 & 0 \\
0 & 0
\end{array}\right.
$$

where $\tilde{c}_{\sigma k}^{\dagger}$ is now the creation operator for the pseudospin $\sigma$, obtained by rotating the original full spin-3/2 basis of Eq. (9) into the band basis and then projecting the effect of the higher energy bands on the low-energy bands. In this new basis the Green's function can be decomposed as

$$
\mathcal{G}=\left(\begin{array}{cc}
\mathcal{G}_{1} & 0 \\
0 & \mathcal{G}_{2}
\end{array}\right)
$$

where

$$
\begin{aligned}
\mathcal{G}_{1}= & \frac{1}{\left[\omega-h_{z}(\boldsymbol{k})\right]^{2}-\tilde{\varepsilon}_{\boldsymbol{k}}^{2}-\psi_{-}^{2}(\boldsymbol{k})} \\
& \times\left(\begin{array}{cc}
\omega+\tilde{\varepsilon}_{\boldsymbol{k}}-h_{z}(\boldsymbol{k}) & \psi_{-}(\boldsymbol{k}) \\
\psi_{-}(\boldsymbol{k}) & \omega-\tilde{\varepsilon}_{\boldsymbol{k}}-h_{z}(\boldsymbol{k})
\end{array}\right)
\end{aligned}
$$$$
\left.\begin{array}{cc}
0 & 0 \\
0 & 0 \\
\tilde{\varepsilon}_{\boldsymbol{k}}-h_{z}(\boldsymbol{k}) & -\psi_{-}(\boldsymbol{k}) \\
-\psi_{-}(\boldsymbol{k}) & -\tilde{\varepsilon}_{\boldsymbol{k}}-h_{z}(\boldsymbol{k})
\end{array}\right),
$$

$\left.\sqrt{3} \beta k_{y} k_{z}, \sqrt{3} \beta k_{x} k_{z}, \frac{\sqrt{3} \beta}{2}\left(k_{x}^{2}-k_{y}^{2}\right), \beta\left(k_{z}^{2}-\frac{k_{x}^{2}+k_{y}^{2}}{2}\right)\right\}$. As the chemical potential is usually found in one of the normal-state bands and assuming that the important physical properties of the system are then also coming from that band, we can extract an effective Hamiltonian for this band (here the " $\varepsilon_{-}$" band). The whole BdG Hamiltonian then simplifies into the form $[39,40]$

$$
\hat{\mathcal{H}}_{b-}(\boldsymbol{k})=\left(\begin{array}{cc}
\tilde{\varepsilon}(\boldsymbol{k}) \sigma_{0}+h_{z}(\boldsymbol{k}) \sigma_{z} & \psi_{-}(\boldsymbol{k}) i \sigma_{y} \\
-\psi_{-}^{*}(\boldsymbol{k}) i \sigma_{y} & -\tilde{\varepsilon}(\boldsymbol{k}) \sigma_{0}-h_{z}(\boldsymbol{k}) \sigma_{z}
\end{array}\right),
$$

where

$$
\begin{gathered}
\tilde{\varepsilon}(\boldsymbol{k})=\varepsilon_{-}(\boldsymbol{k})+\zeta(\boldsymbol{k}), \\
\psi_{-}(\boldsymbol{k})=\Delta_{1} \psi_{\boldsymbol{k}}-\Delta_{0} \frac{\varepsilon_{3}+i \varepsilon_{2}}{\left|\boldsymbol{\varepsilon}_{\boldsymbol{k}}\right|}
\end{gathered}
$$

with

$$
\begin{aligned}
\zeta(\boldsymbol{k}) & =\frac{\left|\Delta_{0}\right|^{2}}{4\left|\boldsymbol{\varepsilon}^{\prime}(\boldsymbol{k})\right|^{3}}\left(4\left|\boldsymbol{\varepsilon}^{\prime}(\boldsymbol{k})\right|^{2}-2 \varepsilon_{2}^{2}-2 \varepsilon_{3}^{2}\right), \\
h_{z}(\boldsymbol{k}) & =\frac{\left|\Delta_{0}\right|^{2}}{\left|\boldsymbol{\varepsilon}^{\prime}(\boldsymbol{k})\right|^{2}} \sqrt{\varepsilon_{1}^{2}+\varepsilon_{4}^{2}+\varepsilon_{5}^{2}}
\end{aligned}
$$

and energy dispersion given by

$$
E= \pm h_{z}(\boldsymbol{k}) \pm \sqrt{\tilde{\varepsilon}(\boldsymbol{k})^{2}+\left|\psi_{-}(\boldsymbol{k})\right|^{2}}
$$

This is thus a psuedospin description of the low-energy physics, which is equivalent to the low-energy basis of the original spin-3/2 system, where we modified the lower energy band by the effect of the higher energy band. In this effective low-energy model, the normal band $\varepsilon_{-}(\boldsymbol{k})$ is modified by the $\zeta(\boldsymbol{k})$ term and a momentum dependent pseudomagnetic field $h_{z}(\boldsymbol{k})$. Moreover, $\psi_{-}(\boldsymbol{k})$ is the effective nodal superconducting gap potential with a chiral $d$-wave form. It produces a nodal ring on the $k_{z}=0$ plane, while a finite pseudomagnetic field $h_{z}(\boldsymbol{k})$ inflates it into a BFS [40].

As the pseudomagnetic field is in the $z$ direction, Eq. (23) can be further simplified by rearranging the basis to $\left(\tilde{c}_{\uparrow k}^{\dagger} \tilde{c}_{\downarrow-k} \tilde{c}_{\downarrow k}^{\dagger} \tilde{c}_{\uparrow-k}\right)$, resulting in the BdG Hamiltonian

with the Green's function of the other block, $\mathcal{G}_{2}$, obtained simply by changing $\left[h_{z}(\boldsymbol{k}), \psi_{-}(\boldsymbol{k})\right] \rightarrow-\left[h_{z}(\boldsymbol{k}), \psi_{-}(\boldsymbol{k})\right]$. Finally, we can extract the anomalous Green's function as the first block $\mathcal{G}_{1}$ giving

$$
\mathcal{F}_{1}=\psi_{-}(\boldsymbol{k}) \frac{\omega^{2}+h_{z}^{2}(\boldsymbol{k})-\tilde{\varepsilon}_{\boldsymbol{k}}^{2}-\psi_{-}^{2}(\boldsymbol{k})+2 \omega h_{z}(\boldsymbol{k})}{\left[\omega^{2}+h_{z}^{2}(\boldsymbol{k})-\tilde{\varepsilon}_{\boldsymbol{k}}^{2}-\psi_{-}^{2}(\boldsymbol{k})\right]^{2}-4 \omega^{2} h_{z}^{2}(\boldsymbol{k})},
$$

where we now easily find the odd- $\omega$ component as

$$
\mathcal{F}_{1}^{T o}=\psi_{-}(\boldsymbol{k}) \frac{2 \omega h_{z}(\boldsymbol{k})}{\left[\omega^{2}+h_{z}^{2}(\boldsymbol{k})-\tilde{\varepsilon}_{\boldsymbol{k}}^{2}-\psi_{-}^{2}(\boldsymbol{k})\right]^{2}-4 \omega^{2} h_{z}^{2}(\boldsymbol{k})} .
$$


This result clearly illustrates how the odd- $\omega$ pair amplitude is directly proportional to the pseudomagnetic field $h_{z}(\boldsymbol{k})$. At the same time, $h_{z}(\boldsymbol{k})$ is also the term explicitly responsible for the appearance of BFS. Thus, for this low-energy effective band model, we find that odd- $\omega$ pairing and BFS always exist together. This also confirms analytically our numerical results in Sec. III B 3.

\section{SUMMARY AND CONCLUSIONS}

To summarize, we have introduced the $\mathcal{J P} \mathcal{T}=-1$ symmetry classification for superconductivity in spin-3/2 fermion systems, where the Cooper pairs are composed of two electrons with finite angular momenta $j_{i}=3 / 2$. Here, $\mathcal{J}, \mathcal{P}$, and $\mathcal{T}$ are the symmetry operators for the $z$ component of the total angular momentum, spatial parity, and time (or equivalently, frequency), respectively. In addition to spin-singlet and spintriplet pairing found in conventional spin-1/2 systems, there are here two additional spin structures, namely, spin-quintet and spin-septet Cooper pairs or equivalently pair amplitudes. All of these Cooper pairs can further be classified into evenand odd-spatial parity as well as even- and odd-frequency pairing. Following the antisymmetry condition $\mathcal{J P} \mathcal{T}=-1$, this generates a total of 32 different classes of superconducting pair symmetries.

To illustrate the existence of the different pair symmetry classes, we have also studied a model suitable to describe the superconducting half-Heusler compounds hosting low-energy spin-3/2 fermions, using several different superconducting pair potentials suggested in the current literature; even-parity $s$-wave, odd-parity $p$-wave, and chiral even-parity $d$-wave pair potentials. By calculating the anomalous Green's function we have numerically accessed the superconducting pair amplitudes for all cases and showed how the different classes can be present, including large odd- $\omega$ pair amplitudes. In particular, we have found that the chiral $d$-wave pair potential generates large spin-septet even-parity odd- $\omega$ pair amplitudes.

We have also derived a general analytical expression for the odd- $\omega$ pair amplitude, applicable to any spin-3/2 superconductor as well as other multiband systems. Using this expression we have investigated the relation between the odd$\omega$ pair amplitude and the appearance of a BFS. We have found that both BFS and odd- $\omega$ pairing are always present together for superconducting pair potentials with an odd-gap time-reversal product and noncommuting with the normalstate Hamiltonian. Using a minimal low-energy model of a spin-3/2 superconductor with a possible BFS, we have further been able to sharpen this criteria of coexistence of BFS and odd- $\omega$ pairing and shown that a BFS and a finite odd- $\omega$ pair amplitude are always appearing simultaneously in this effective model. Based on these results, we speculate that any superconductor with a BFS hosts finite odd- $\omega$ pair amplitudes, albeit the reverse is already known to not be true.

Note added. Recently, two other works appeared discussing various aspects of odd- $\omega$ pairing in spin-3/2 superconductors. In Ref. [62] a finite odd- $\omega$ pair amplitude was found to favor a $\pi$ state in a Josephson junction consisting of two spin-3/2 superconductors. However, this work neither classified the different superconducting pair symmetries, including the different possibilities of odd- $\omega$ pair amplitudes, nor considered the relation between odd- $\omega$ pairs and BFSs. In Ref. [63] pairing of the bogolons living on the BFS was shown to be odd- $\omega$ in nature. This is, however, very different from our work as it considers pairing within the BFS, while we consider pairing of the original spin-3/2 fermions (which then can generate the BFS). Thus both of these works are very different from our results.

\section{ACKNOWLEDGMENTS}

We thank D. Chakraborty for technical help and acknowledge financial support from the European Research Council (ERC) under the European Unions Horizon 2020 research and innovation program (ERC-2017-StG-757553), the Knut and Alice Wallenberg Foundation through the Wallenberg Academy Fellows program, and the Swedish Research Council (Vetenskapsrådet Grant No. 2018-03488).

\section{APPENDIX: MATRIX OPERATORS}

The angular momentum operators for spin $j=3 / 2$ fermions are expressed in matrix form as

$$
\begin{gathered}
\hat{J}_{x}=\frac{1}{2}\left(\begin{array}{cccc}
0 & \sqrt{3} & 0 & 0 \\
\sqrt{3} & 0 & 2 & 0 \\
0 & 2 & 0 & \sqrt{3} \\
0 & 0 & \sqrt{3} & 0
\end{array}\right), \\
\hat{J}_{y}=\frac{i}{2}\left(\begin{array}{cccc}
0 & -\sqrt{3} & 0 & 0 \\
\sqrt{3} & 0 & -2 & 0 \\
0 & 2 & 0 & -\sqrt{3} \\
0 & 0 & \sqrt{3} & 0
\end{array}\right), \text { and } \\
\hat{J}_{z}=\frac{1}{2}\left(\begin{array}{cccc}
3 & 0 & 0 & 0 \\
0 & 1 & 0 & 0 \\
0 & 0 & -1 & 0 \\
0 & 0 & 0 & -3
\end{array}\right) .
\end{gathered}
$$

The unitary operator is defined as

$$
U_{T}=\left(\begin{array}{cccc}
0 & 0 & 0 & 1 \\
0 & 0 & -1 & 0 \\
0 & 1 & 0 & 0 \\
-1 & 0 & 0 & 0
\end{array}\right)
$$

[1] J. Linder and A. V. Balatsky, Odd-frequency superconductivity, Rev. Mod. Phys. 91, 045005 (2019).

[2] Y. Tanaka, M. Sato, and N. Nagaosa, Symmetry and topology in superconductors-Odd-frequency pairing and edge states-, J. Phys. Soc. Jpn. 81, 011013 (2012).
[3] A. Balatsky and E. Abrahams, New class of singlet superconductors which break the time reversal and parity, Phys. Rev. B 45, 13125 (1992).

[4] V. L. Berezinskii, New model of the anisotropic phase of superfluid $\mathrm{He}^{3}$, Pis'ma Zh. Eksp. Teor. Fiz. 20, 628 (1974). 
[5] A. F. Volkov, F. S. Bergeret, and K. B. Efetov, Odd Triplet Superconductivity in Superconductor-Ferromagnet Multilayered Structures, Phys. Rev. Lett. 90, 117006 (2003).

[6] Y. Tanaka, Y. Tanuma, and A. A. Golubov, Odd-frequency pairing in normal-metal/superconductor junctions, Phys. Rev. B 76, 054522 (2007).

[7] T. Yokoyama, Y. Tanaka, and A. A. Golubov, Manifestation of the odd-frequency spin-triplet pairing state in diffusive ferromagnet/superconductor junctions, Phys. Rev. B 75, 134510 (2007).

[8] M. Eschrig and T. Löfwander, Triplet supercurrents in clean and disordered half-metallic ferromagnets, Nat. Phys. 4, 138 (2008).

[9] F. Parhizgar and A. M. Black-Schaffer, Unconventional proximity-induced superconductivity in bilayer systems, Phys. Rev. B 90, 184517 (2014).

[10] P. Dutta, K. R. Alves, and A. M. Black-Schaffer, Thermoelectricity carried by proximity-induced odd-frequency pairing in ferromagnet/superconductor junctions, Phys. Rev. B 102, 094513 (2020).

[11] A. M. Black-Schaffer and A. V. Balatsky, Odd-frequency superconducting pairing in multiband superconductors, Phys. Rev. B 88, 104514 (2013).

[12] C. Triola, J. Cayao, and A. M. Black-Schaffer, The role of oddfrequency pairing in multiband superconductors, Ann. Phys. 532, 1900298 (2020).

[13] F. Parhizgar and A. M. Black-Schaffer, Diamagnetic and paramagnetic Meissner effect from odd-frequency pairing in multiorbital superconductors, Phys. Rev. B 104, 054507 (2021).

[14] A. M. Black-Schaffer and A. V. Balatsky, Odd-frequency superconducting pairing in topological insulators, Phys. Rev. B 86, 144506 (2012).

[15] F. Crépin, P. Burset, and B. Trauzettel, Odd-frequency triplet superconductivity at the helical edge of a topological insulator, Phys. Rev. B 92, 100507(R) (2015).

[16] J. Cayao and A. M. Black-Schaffer, Odd-frequency superconducting pairing and subgap density of states at the edge of a two-dimensional topological insulator without magnetism, Phys. Rev. B 96, 155426 (2017).

[17] P. Dutta and A. M. Black-Schaffer, Signature of odd-frequency equal-spin triplet pairing in the Josephson current on the surface of Weyl nodal loop semimetals, Phys. Rev. B 100, 104511 (2019).

[18] J. Schmidt, F. Parhizgar, and A. M. Black-Schaffer, Oddfrequency superconductivity and Meissner effect in the doped topological insulator $\mathrm{Bi}_{2} \mathrm{Se}_{3}$, Phys. Rev. B 101, 180512(R) (2020).

[19] P. Dutta, F. Parhizgar, and A. M. Black-Schaffer, Finite bulk Josephson currents and chirality blockade removal from interorbital pairing in magnetic Weyl semimetals, Phys. Rev. B 101, 064514 (2020).

[20] F. Parhizgar and A. M. Black-Schaffer, Large Josephson current in Weyl nodal loop semimetals due to odd-frequency superconductivity, npj Quantum. Mater. 5, 42 (2020).

[21] P. Coleman, E. Miranda, and A. Tsvelik, Possible Realization of Odd-Frequency Pairing in Heavy Fermion Compounds, Phys. Rev. Lett. 70, 2960 (1993).

[22] P. Coleman, E. Miranda, and A. Tsvelik, Odd-frequency pairing in the Kondo lattice, Phys. Rev. B 49, 8955 (1994).
[23] C. Triola and A. M. Black-Schaffer, Odd-frequency pairing and Kerr effect in the heavy-fermion superconductor $\mathrm{UPt}_{3}$, Phys. Rev. B 97, 064505 (2018).

[24] C. Triola and A. V. Balatsky, Odd-frequency superconductivity in driven systems, Phys. Rev. B 94, 094518 (2016).

[25] J. Cayao, C. Triola, and A. M. Black-Schaffer, Floquet engineering bulk odd-frequency superconducting pairs, Phys. Rev. B 103, 104505 (2021).

[26] A. Di Bernardo, S. Diesch, Y. Gu, J. Linder, G. Divitini, C. Ducati, E. Scheer, M. G. Blamire, and J. W. A. Robinson, Signature of magnetic-dependent gapless odd frequency states at superconductor/ferromagnet interfaces, Nat. Commun. 6, 8053 (2015).

[27] A. Di Bernardo, Z. Salman, X. L. Wang, M. Amado, M. Egilmez, M. G. Flokstra, A. Suter, S. L. Lee, J. H. Zhao, T. Prokscha, E. Morenzoni, M. G. Blamire, J. Linder, and J. W. A. Robinson, Intrinsic Paramagnetic Meissner Effect Due to $s$-Wave Odd-Frequency Superconductivity, Phys. Rev. X 5, 041021 (2015).

[28] R. S. Keizer, S. T. B. Goennenwein, T. M. Klapwijk, G. Miao, G. Xiao, and A. Gupta, A spin triplet supercurrent through the half-metallic ferromagnet $\mathrm{CrO}_{2}$, Nature (London) 439, 825 (2006).

[29] T. S. Khaire, M. A. Khasawneh, W. P. Pratt, and N. O. Birge, Observation of Spin-Triplet Superconductivity in Co-Based Josephson Junctions, Phys. Rev. Lett. 104, 137002 (2010).

[30] J. W. A. Robinson, J. D. S. Witt, and M. G. Blamire, Controlled injection of spin-triplet supercurrents into a strong ferromagnet, Science 329, 59 (2010).

[31] J. A. Krieger, A. Pertsova, S. R. Giblin, M. Döbeli, T. Prokscha, C. W. Schneider, A. Suter, T. Hesjedal, A. V. Balatsky, and Z. Salman, Proximity-Induced Odd-Frequency Superconductivity in a Topological Insulator, Phys. Rev. Lett. 125, 026802 (2020).

[32] P. M. R. Brydon, L. Wang, M. Weinert, and D. F. Agterberg, Pairing of $j=3 / 2$ Fermions in Half-Heusler Superconductors, Phys. Rev. Lett. 116, 177001 (2016).

[33] H. Kim, K. Wang, Y. Nakajima, R. Hu, S. Ziemak, P. Syers, L. Wang, H. Hodovanets, J. D. Denlinger, P. M. R. Brydon, D. F. Agterberg, M. A. Tanatar, R. Prozorov, and J. Paglione, Beyond triplet: Unconventional superconductivity in a spin-3/2 topological semimetal, Sci. Adv. 4, eaao4513 (2018).

[34] T. V. Bay, T. Naka, Y. K. Huang, and A. de Visser, Superconductivity in noncentrosymmetric yptbi under pressure, Phys. Rev. B 86, 064515 (2012).

[35] J. R. Schrieffer, Theory of Superconductivity (CRC Press, Boca Raton, FL, 2018).

[36] J. W. F. Venderbos, L. Savary, J. Ruhman, P. A. Lee, and L. Fu, Pairing States of Spin- $\frac{3}{2}$ Fermions: SymmetryEnforced Topological Gap Functions, Phys. Rev. X 8, 011029 (2018).

[37] J. Yu and C.-X. Liu, Singlet-quintet mixing in spin-orbit coupled superconductors with $j=\frac{3}{2}$ fermions, Phys. Rev. B 98, 104514 (2018).

[38] B. Roy, S. A. A. Ghorashi, M. S. Foster, and A. H Nevidomskyy, Topological superconductivity of spin-3/2 carriers in a three-dimensional doped Luttinger semimetal, Phys. Rev. B 99, 054505 (2019).

[39] D. F. Agterberg, P. M. R. Brydon, and C. Timm, Bogoliubov Fermi Surfaces in Superconductors with Broken Time-Reversal Symmetry, Phys. Rev. Lett. 118, 127001 (2017). 
[40] P. M. R. Brydon, D. F. Agterberg, H. Menke, and C. Timm, Bogoliubov Fermi surfaces: General theory, magnetic order, and topology, Phys. Rev. B 98, 224509 (2018).

[41] H. Menke, C. Timm, and P. M. R. Brydon, Bogoliubov Fermi surfaces stabilized by spin-orbit coupling, Phys. Rev. B 100, 224505 (2019).

[42] J. M. Link, I. Boettcher, and I. F. Herbut, $d$-wave superconductivity and Bogoliubov-Fermi surfaces in Rarita-SchwingerWeyl semimetals, Phys. Rev. B 101, 184503 (2020).

[43] L. Fu, Odd-parity topological superconductor with nematic order: Application to $\mathrm{Cu}_{x} \mathrm{Bi}_{2} \mathrm{Se}_{3}$, Phys. Rev. B 90, 100509(R) (2014).

[44] Y. Gao, W.-P. Su, and J.-X. Zhu, Interorbital pairing and its physical consequences for iron pnictide superconductors, Phys. Rev. B 81, 104504 (2010).

[45] C. Setty, S. Bhattacharyya, Y. Cao, A. Kreisel, and P. J. Hirschfeld, Topological ultranodal pair states in iron-based superconductors, Nat. Commun. 11, 523 (2020).

[46] F. F. Tafti, T. Fujii, A. Juneau-Fecteau, S. René de Cotret, N. Doiron-Leyraud, A. Asamitsu, and L. Taillefer, Superconductivity in the noncentrosymmetric half-Heusler compound LuPtBi: A candidate for topological superconductivity, Phys. Rev. B 87, 184504 (2013).

[47] Y. Nakajima, R. Hu, K. Kirshenbaum, A. Hughes, P. Syers, X. Wang, K. Wang, R. Wang, S. R. Saha, D. Pratt et al., Topological RPdBi half-Heusler semimetals: A new family of noncentrosymmetric magnetic superconductors, Sci. Adv. 1, e1500242 (2015).

[48] H. Xiao, T. Hu, W. Liu, Y. L. Zhu, P. G. Li, G. Mu, J. Su, K. Li, and Z. Q. Mao, Superconductivity in the half-Heusler compound TbPdBi, Phys. Rev. B 97, 224511 (2018).

[49] T. Kawakami, T. Okamura, S. Kobayashi, and M. Sato, Topological Crystalline Materials of $j=3 / 2$ Electrons: Antiperovskites, Dirac Points, and High Winding Topological Superconductivity, Phys. Rev. X 8, 041026 (2018).

[50] J. M. Link and I. F. Herbut, Bogoliubov-Fermi Surfaces in Noncentrosymmetric Multicomponent Superconductors, Phys. Rev. Lett. 125, 237004 (2020).
[51] I. F. Herbut and J. M. Link, Bogoliubov-Fermi surface with inversion symmetry and electron-electron interactions: Relativistic analogies and lattice theory, Phys. Rev. B 103, 144517 (2021).

[52] J. Linder, A. Sudbø, T. Yokoyama, R. Grein, and M. Eschrig, Signature of odd-frequency pairing correlations induced by a magnetic interface, Phys. Rev. B 81, 214504 (2010).

[53] G. D. Mahan, Many-Particle Physics (Springer Science \& Business Media, New York, 2013).

[54] D. J. Griffiths, Introduction to Quantum Mechanics (Prentice Hall, Englewood Cliffs, NJ, 2010).

[55] L. Savary, J. Ruhman, J. W. F. Venderbos, L. Fu, and P. A. Lee, Superconductivity in three-dimensional spin-orbit coupled semimetals, Phys. Rev. B 96, 214514 (2017).

[56] W. Yang, Y. Li, and C. Wu, Topological Septet Pairing with Spin- $\frac{3}{2}$ Fermions: High-Partial-Wave Channel Counterpart of the ${ }^{3} \mathrm{He}-B$ phase, Phys. Rev. Lett. 117, 075301 (2016).

[57] J. M. Luttinger and W. Kohn, Motion of Electrons and Holes in Perturbed Periodic Fields, Phys. Rev. 97, 869 (1955).

[58] I. Boettcher and I. F. Herbut, Superconducting quantum criticality in three-dimensional Luttinger semimetals, Phys. Rev. B 93, 205138 (2016).

[59] I. Boettcher and I. F. Herbut, Unconventional Superconductivity in Luttinger Semimetals: Theory of Complex Tensor Order and the Emergence of the Uniaxial Nematic State, Phys. Rev. Lett. 120, 057002 (2018).

[60] A. Ramires and M. Sigrist, Identifying detrimental effects for multiorbital superconductivity: Application to $\mathrm{Sr}_{2} \mathrm{RuO}_{4}$, Phys. Rev. B 94, 104501 (2016).

[61] A. Ramires, D. F. Agterberg, and M. Sigrist, Tailoring $T_{c}$ by symmetry principles: The concept of superconducting fitness, Phys. Rev. B 98, 024501 (2018).

[62] D. Kim, S. Kobayashi, and Y. Asano, Josephson effect of superconductors with $j=3 / 2$ electrons, Phys. Rev. B 103, 184516 (2021).

[63] T. Miki, S.-T. Tamura, S. Iimura, and S. Hoshino, Oddfrequency pairing inherent in Bogoliubov Fermi liquid, arXiv:2103.02251. 